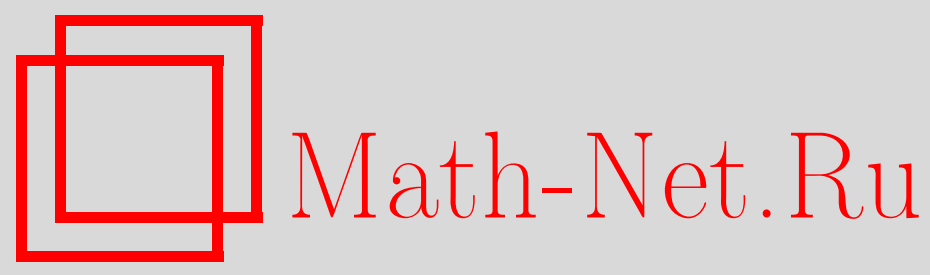

В. К. Захаров, Локальная теория множеств, Матем. заметки, 2005, том 77, выпуск 2, 194-212

DOI: https://doi.org/10.4213/mzm2490

Использование Общероссийского математического портала Math-Net.Ru подразумевает, что вы прочитали и согласны с пользовательским соглашением http://www.mathnet.ru/rus/agreement

Параметры загрузки:

IP : 18.209.158.208

26 апреля 2023 г., 13:07:50

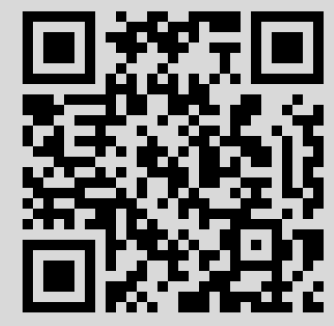




\title{
ЛОКАЛЬНАЯ ТЕОРИЯ МНОЖЕСТВ
}

\author{
B. К. Захаров
}

В 1945 г. Эйленбергом и Маклейном было введено новое математическое понятие категории. Однако с самого начала своего возникновения теория категорий столкнулась с тем неприятным обстоятельством, что она не вмещалась не только в рамки теории множеств в аксиоматике Цермело-Френкеля, но даже и в рамки теории классов и множеств в аксиоматике фон Неймана-Бернайса-Гёделя. По этой причине в 1959 г. Маклейн поставил общую проблему построения новой и более гибкой аксиоматической теории множеств, которая могла бы служить адекватньм логическим основанием для всей наивной теории категорий.

В данной работе даются аксиоматические основания локальной теории множеств. Эта теория может быть одним из возможных вариантов решения проблемы Маклейна.

Библиография: 19 названий.

1. Введение. Кризис, возникший в наивной теории множеств в начале $\mathrm{XX}$ века, привел к возникновению нескольких строгих аксиоматических построений теорий математических совокупностей. Наиболее широко используемыми из них являются теория множеств в аксиоматике Цермело-Френкеля $(Z F)$ (см. [1]) и теория классов и множсеств в аксиоматике фон Неймана-Бернайса-Гёделя (NBG) (см. [2] и [3]).

Эти аксиоматические теории устранили все известные парадоксы наивной теории множеств за счет резкого ограничения возможных выразительных средств. В то же время они давали возможность включать почти все существовавшие тогда математические объекты и конструкции в рамки этих теорий.

Однако уже в 1945 году в основополагающей работе [4] Эйленбергом и Маклейном было введено новое математическое понятие категории. С самого начала своего возникновения теория категорий столкнулась с тем неприятньм обстоятельством, что она не вмешалась ни в рамки $Z F$, ни в рамки $N B G$ (см. [4]). Поэтому Маклейном в работе [5] 1959 года была поставлена общая проблема построения новой и более гибкой аксиоматической теории множеств, которая могла бь служить адекватным логическим основанием для всей наивной теории категорий.

С тех пор было предложено несколько теорий множеств в качестве оснований для теории категорий (см. [6]-[16]). Однако либо они не обслуживали всей теории категорий [12]-[16], либо были слишком сильны для теории категорий [6]-[9], либо были слишком сложны [10], [11]. Наиболее широкую известность приобрела теория множеств $Z F+U$ с

Работа выполнена при частичной поддержке Российского фонда фундаментальных исследований, грант № 02-01-00-218, и программы “Университеты России”. 
аксиомой универсальности $U$, принадлежащей Эресманну, Дедекеру, Зоннеру и Гротендику и утверждающей, что каждое множество принадлежит некоторому универсальному множеству, которое замкнуто относительно основных теоретико-множественных конструкций и содержит $\omega$ в качестве элемента. Аксиома универсальности $U$ равносильна аксиоме недостижимости $I$, согласно которой для любого порядкового числа $\alpha$ существует строго большее (сильно) недостижимое кардинальное число $\varkappa$. Теория $Z F+U$ дает очень простое решение проблемы. Однако аксиома $U$ является слишком сильной и излишней для потребностей теории категорий, поскольку она влечет существование трансфинитной коллекции $\left(U_{\alpha} \mid \alpha \in \mathbf{O n}\right)$ универсальных множеств $U_{\alpha}$, индексированной всеми ординалами $\alpha$, в то время как для потребностей теории категорий (для формализации операций “категория категорий” и “категория функторов”) достаточно иметь только счетную коллекцию $\left(U_{n} \mid n \in \omega\right)$ универсальных множеств, как это сделано у да Косты.

Более слабая, чем $Z F+U$, теория $Z F+U(\omega)$ с аксиомой $\omega$-универсальности $U(\omega)$, утверждающей существование счетной совокупности $\left(U_{n} \mid n \in \omega\right)$ универсальных множеств, обеспечивает все потребности теории категорий, однако она оставляет за пределами категорного рассмотрения те объекты, которые не являются элементами универсумов $U_{n}$, поскольку приводит к потере важнейшего свойства универсального охвата

$$
\forall x \quad \exists U \quad(x \in U \wedge U \text { универсально }) .
$$

Именно эта неадекватность теорий $Z F+U$ и $Z F+U(\omega)$ и стимулировала все дальнейшие исследования по проблеме Маклейна. Необходимо было построить аксиоматическую теорию множеств $S$, которая

1) имеет свойство универсального охвата;

2) имеет перечислимую метасовокупность всех универсумов.

Теории $Z F+U$ и $Z F+U(\omega)$ не обладают этими двумя свойствами сразу. Теории множеств, введенные да Костой [10], [11], обладают свойствами 1) и 2), однако они не являются теориями первого порядка, поскольку используют неконструктивноеправило вывода Карнапа и некоторые свойства натуральных чисел.

В 2000 г. автором статьи в качестве более адекватного основания для теории категорий была предложена локальная теория множеств (LTS) (см. анонсы в [17] и [18]). Основная идея $L T S$, отличающая ее от теорий $Z F+U$ и $Z F+U(\omega)$, состоит в отказе от задания глобальной теоретико-множественной структуры и в задании только ее локальных вариантов.

При исследовании $L T S$ было установлено, что глобальная аксиомная схема замещения не вьводится в $L T S$. Это показывает, что $L T S$ не является вариантом теорий $Z F+U$ и $Z F+U(\omega)$, и $L T S$ строго слабее теории $Z F+U$. Кроме того, было установлено, что непротиворечивость каждой из теорий $Z F+U$ и $Z F+U(\omega)$ влечет непротиворечивость $L T S$. Это значит, что $L T S$ более надежна, чем эти теории.

Более того, было доказано, что в $L T S$ не вьводится аксиома $\omega$-универсальности. Поэтому в 2003 г. автором статьи в качестве равнонепротиворечивого усиления $L T S$ была введена локально минимальная теория множеств (LMTS), получающаяся прибавлением к $L T S$ отрицания аксиомы $\omega$-универсальности. Следовательно, $L M T S$ обладает указанными вьше свойствами 1) и 2). Таким образом, $L M T S$ является более естественной для теории категорий, чем теории $Z F+U$ и $Z F+U(\omega)$. 
В данной статье приводятся собственные аксиомы и аксиомные схемы $L T S$ и $L M T S$ и определяются важнейшие теоретико-множественные конструкции. Для подтверждения того, что $L T S$ может служить адекватньм логическим основанием для теории категорий, в рамках $L T S$ формализуются основные категорные понятия и доказьваются теоремы, утверждающие необходимую замкнутость этих понятий относительно таких важнейших операций как “категория категорий” и “категория функторов".

По причине ограниченности объема статьи другие указанные выше результаты для $L T S$ и $L M T S$ в данную статью не включены и будут опубликованы в отдельных работах.

Автор выражает глубокую благодарность С.И. Адяну, Н. К. Верещагину, В. Г. Кановею, В. А. Любецкому, А. В. Михалеву и А. А. Яшину за интерес к работе и рядценных консультаций.

2. Математические аксиомы и аксиомные схемы локальной теории множеств и локально-минимальной теории множеств. Предлагаемые теории являются теориями первого порядка (см. [3], [19]). Имеются следующие спеииальные символы: $\in, \bowtie, \varnothing, \mathfrak{a}$. Символы $\varnothing$ и а обозначают постоянные. Буквы $x, X, y, Y, z, Z, u, U$, $v, V, w, W$ с любым количеством штрихов обозначают переменные. Функииональны символов нет. Имеются два предикатных символа: унарньй $\bowtie$ и бинарньй $\in$.

Термы и формулы определяются обычным для теории первого порядка образом (см. [3], [19]). Термы в рассматриваемом случае - это постоянные и переменные. Элементарные формулы имеют вид $A \bowtie$ и $A \in B$, где $A$ и $B$ - термы.

Объекты данных теорий именуются классами.

Класс $A$ назовем подклассом класса $B$ (в обозначении $A \subset B$ ), если

$$
\forall x(x \in A \Longrightarrow x \in B) .
$$

Классы назьваются равными (в обозначении $A=B)$, если $(A \subset B) \wedge(B \subset A)$. Это равенство подчиняется следующей аксиоме.

A1 (Аксиома объемности). Выполнено

$$
\forall y \forall z((y=z) \Longrightarrow(\forall X(y \in X \Longleftrightarrow z \in X))) .
$$

Пусть $\alpha$ - некоторый фиксированньй класс. Класс $A$ назовем классом класса $\alpha$ (三 $\alpha$-классом), если $A \subset \alpha$. Класс $A$ назовем множеством класса $\alpha$ (三 $\alpha$-множеством), если $A \in \alpha$.

Следующая схема аксиом постулирует существование $\alpha$-классов.

Формула $\varphi$ называется $\alpha$-предикативной (см. [3, гл. $4, \S 1]$ ), если для любых переменных $x$ все знакосочетания $\forall x$ и $\exists x$, входящие в формулу $\varphi$, располагаются только в позициях следующих видов:

$$
\forall x(x \in \alpha \Longrightarrow \ldots) \quad \text { и } \quad \exists x(x \in \alpha \wedge \ldots) .
$$

AS2 (Аксиомная схема полного охвата). Пусть $\varphi(x)$ - $X$-предикативная формула такая, что подстановка $\varphi(x \| y)$ допустима. Тогда

$$
\forall X \exists Z(\forall y((y \in Z) \Longleftrightarrow(y \in X \wedge \varphi(y)))) .
$$

Отсюда и из аксиомы объемности следует, что соответствующий $\alpha$-класс единственен. Будем его обозначать через $\{x \in \alpha \mid \varphi(x)\}$. 
А3 (Аксиома пустого класса). Вьполнено

$$
\forall Z((\forall x(x \notin Z)) \Longleftrightarrow(Z=\varnothing))
$$

Лемма 1. Имеет место утверждение $\forall X(\varnothing \subset X)$.

ДоказАтЕльство. Формулы $x \in \varnothing$ и $x \in X$ обозначим через $\varphi$ и $\psi$ соответственно. Применим логическую теорему $\neg \varphi \Longrightarrow(\varphi \Longrightarrow \psi)$. Из А3 следует $\neg \varphi$. Отсюда по правилу импликации следует $\varphi \Rightarrow \psi$. Применяя правило обобщения, получаем $\forall x(x \in \varnothing \Longrightarrow x \in X)$.

Класс $\alpha$ назовем универсальным, если $\alpha \bowtie$.

А4 (Аксиома равноуниверсальности). Выполнено

$$
\forall U \forall V((U=V) \Longrightarrow(U \bowtie \Longleftrightarrow V \bowtie)) .
$$

Эта аксиома постулирует, что равные классы являются равноуниверсальными.

А5 (Аксиома инфра-универсальности). Вьполнено

$$
(\mathfrak{a} \bowtie) \wedge(\forall U(U \bowtie \Longrightarrow \mathfrak{a} \subset U)) \text {. }
$$

Эта аксиома постулирует, что класс а является “наименьшим" универсальньп классом. Назовем его инфра-универсальным или инфра-универсумом.

А6 (Аксиома универсальности). Выполнено

$$
\forall X \exists U(U \bowtie \wedge X \in U) .
$$

Эта аксиома постулирует, что каждый класс $A$ является элементом некоторого универсального класса $\alpha$.

Последующие аксиомы разъясняют значение понятия универсальности.

А7 (Аксиома транзитивности). Вьполнено

$$
\forall U(U \bowtie \Longrightarrow \forall X(X \in U \Longrightarrow X \subset U))
$$

Эта аксиома постулирует, что, если $\alpha$ - универсальньй класс, то каждое $\alpha$-множество является $\alpha$-классом.

А8 (Аксиома подмножества). Выполнено

$$
\forall U(U \bowtie \Longrightarrow \forall X \forall Y(X \in U \wedge Y \subset X \Longrightarrow Y \in U)) .
$$

Эта аксиома постулирует, что если $\alpha$ - универсальный класс, то подкласс $\alpha$-множества является $\alpha$-множеством.

В рамках каждого класса $\alpha$ определим все основные теоретико-множественные конструкции.

Для каждого класса $A \alpha$-класс

$$
\mathscr{P}_{\alpha}(A) \equiv\{x \in \alpha \mid x \subset A\}
$$


А9 (Аксиома полного ансамбля). Вьполнено

$$
\forall U\left(U \bowtie \Longrightarrow \forall X\left(X \in U \Longrightarrow \mathscr{P}_{U}(X) \in U\right)\right)
$$

Эта аксиома постулирует, что если $\alpha$ - универсальный класс и $A-\alpha$-множество, то его полньй $\alpha$-ансамбль $\mathscr{P}_{\alpha}(A)$ является тоже $\alpha$-множеством.

Для классов $A$ и $B \alpha$-класс

$$
A \cup_{\alpha} B \equiv\{x \in \alpha \mid x \in A \vee x \in B\}
$$

назовем $\alpha$-объединением классов $A$ и $B$; $\alpha$-класс

$$
A \cap_{\alpha} B \equiv\{x \in \alpha \mid x \in A \wedge x \in B\}
$$

назовем $\alpha$-пересечением классов $A$ и $B$.

A10 (Аксиома бинарного объединения). Вьполнено

$$
\forall U\left(U \bowtie \Longrightarrow \forall X \forall Y\left(X \in U \wedge Y \in U \Longrightarrow X \cup_{U} Y \in U\right)\right)
$$

Эта аксиома постулирует, что если $\alpha$ - универсальньй класс, то бинарное $\alpha$-объединение $\alpha$-множеств является $\alpha$-множеством. Из А10 и А 8 следует, что то же самое верно и для бинарного $\alpha$-пересечения.

Для класса $A$ рассмотрим одиночныц й $\alpha-\kappa л а с c ~$

$$
\{A\}_{\alpha} \equiv\{x \in \alpha \mid x=A\}
$$

Для $\alpha$-множеств $A$ и $B$ назовем $\alpha$-класс

$$
\{A, B\}_{\alpha} \equiv\{A\}_{\alpha} \cup_{\alpha}\{B\}_{\alpha}
$$

неупорядоченной $\alpha$-парой, а $\alpha$-класс

$$
\langle A, B\rangle_{\alpha} \equiv\left\{\{A\}_{\alpha},\{A, B\}_{\alpha}\right\}_{\alpha}
$$

- координатной $\alpha$-парой.

ЛЕМма 2. Пусть $\alpha-$ универсальный класс и классы а и вами. Тогда $\{a\}_{\alpha},\{b\}_{\alpha},\{a, b\}_{\alpha} u\langle a, b\rangle_{\alpha}$ являются $\alpha$-множествами.

ДокАЗАтЕльство. По А9 $\mathscr{P}_{\alpha}(a) \in \alpha$. Из $\{a\}_{\alpha} \subset \mathscr{P}_{\alpha}(a)$ по А 8 следует, что $\{a\}_{\alpha}$ является $\alpha$-множеством. Аналогично $\{b\}_{\alpha}$ является $\alpha$-множеством.

Из А10 теперь следует, что $\{a, b\}_{\alpha} \in \alpha$. Отсюда и из $\{b\}_{\alpha} \in \alpha$ по доказанному свойству следует, что $\langle a, b\rangle_{\alpha} \in \alpha$.

СлЕДСТВИЕ 1. Пусть $\alpha-$ универсальный класс, $a, a^{\prime}, b, b^{\prime} \in \alpha u\langle a, b\rangle_{\alpha}=\left\langle a^{\prime}, b^{\prime}\right\rangle_{\alpha}$. Тогда $a=a^{\prime} u b=b^{\prime}$. 
ДокАЗАТЕЛЬСТво проводится непосредственным рассмотрением всех возможных случаев (см. [1, гл. $2, \S 3])$.

Для классов $A$ и $B$ назовем $\alpha$-класс

$$
A *_{\alpha} B \equiv\left\{x \in \alpha \mid \exists y \exists z\left(y \in \alpha \wedge y \in A \wedge z \in \alpha \wedge z \in B \wedge x=\langle y, z\rangle_{\alpha}\right)\right\}
$$

координатным $\alpha$-произведением классов $A$ и $B$.

Далее $A$ и $B$ обозначают фиксированные $\alpha$-классы.

$\alpha$-подкласс $u \alpha$-класса $A *_{\alpha} B$ будем назьвать $\alpha$-соответствием из $\alpha$-класса $A$ в $\alpha$-класс $B$ и будем обозначать через $u: A \prec_{\alpha} B$. Формулу $u \subset A *_{\alpha} B$ будем обозначать также через $u \rightleftharpoons A \longrightarrow{ }_{\alpha} B$.

Для $\alpha$-соответствия $u: A \longrightarrow{ }_{\alpha} B$ рассмотрим $\alpha$-классы

$$
\begin{aligned}
\operatorname{dom}_{\alpha} u & \equiv\left\{x \in \alpha \mid x \in A \wedge\left(\exists y\left(y \in B \wedge\langle x, y\rangle_{\alpha} \in u\right)\right)\right\} \\
\operatorname{rng}_{\alpha} u & \equiv\left\{y \in \alpha \mid y \in B \wedge\left(\exists x\left(x \in A \wedge\langle x, y\rangle_{\alpha} \in u\right)\right)\right\}
\end{aligned}
$$

$\alpha$-подкласс

$$
B_{a} \equiv u\langle a\rangle \equiv\left\{y \in \alpha \mid y \in B \wedge\left(\langle a, y\rangle_{\alpha} \in u\right)\right\}
$$

$\alpha$-класса $B$ будем называть $\alpha$-классом значений $\alpha$-соответствия $и$ на әлементе $a \in A ; \alpha$-подкласс

$$
u\left[A^{\prime}\right] \equiv\left\{y \in \alpha \mid y \in B \wedge\left(\exists x\left(x \in A^{\prime} \wedge\langle x, y\rangle_{\alpha} \in u\right)\right)\right\}
$$

$\alpha$-класса $B$ будем назьвать образом подкласса $A^{\prime} \alpha$-класса $A$ относительно $\alpha$-соответствия $u: A \longrightarrow_{\alpha} B$. Ясно, что $u\left[\{a\}_{\alpha}\right]=u\langle a\rangle$ для каждого $a \in A$ и $u[A]=\operatorname{rng}_{\alpha} u$.

Если $u\langle a\rangle$ содержит единственньй элемент $b \in B$ (в том смысле, что $\exists y(y \in B \wedge$ $\left.\left.\left(u\langle a\rangle=\{y\}_{\alpha}\right)\right)\right)$, то этот единственный элемент $b$ назьвается значением $\alpha$-соответствия $u$ на әлементе $a \in A$ и обозначается через $u(a)$ или через $b_{a}$.

$\alpha$-соответствие $u: A \longrightarrow{ }_{\alpha} B$ назовем тотальным, если $\operatorname{dom}_{\alpha} u=A$, и однозначным если $u\langle a\rangle=\{u(a)\}_{\alpha}$ для каждого $a \in \operatorname{dom}_{\alpha} u$.

$\alpha$-соответствие $u: A \longrightarrow_{\alpha} B$ будем называть также (многозначной) $\alpha$-коллекиией $\alpha$-подклассов $B_{a} \alpha$-класса $B$, индексированной $\alpha$-классом $A$. В этом случае класс $u$ и формулу $u \rightleftharpoons A \longrightarrow{ }_{\alpha} B$ будем обозначать также через $\left(B_{a} \subset B \mid a \in A\right)_{\alpha}$ и $u \rightleftharpoons\left(B_{a} \subset B \mid a \in A\right)_{\alpha}$ соответственно.

Тотальное однозначное $\alpha$-соответствие $u: A \prec_{\alpha} B$ называется $\alpha$-отображсением из $\alpha$-класса $A$ в $\alpha$-класс $B$ и обозначается через $u: A \rightarrow_{\alpha} B$. Формула

$$
\left(u \rightleftharpoons A \longrightarrow{ }_{\alpha} B\right) \wedge\left(\operatorname{dom}_{\alpha} u=A\right) \wedge \forall x\left(x \in A \Longrightarrow\left(\exists y\left(y \in B \wedge u\langle x\rangle=\{y\}_{\alpha}\right)\right)\right)
$$

обозначается через $u \rightleftharpoons A \rightarrow{ }_{\alpha} B$.

$\alpha$-отображение $u: A \rightarrow_{\alpha} B$ будем назьвать также простой $\alpha$-коллекиией әлементов $b_{a} \alpha$-класса $B$, индексированной $\alpha$-классом $A$. В этом случае класс $u$ и формулу $u \rightleftharpoons A \rightarrow_{\alpha} B$ будем обозначать также через $\left(b_{a} \in B \mid a \in A\right)_{\alpha}$ и $u \rightleftharpoons\left(b_{a} \in B \mid a \in A\right)_{\alpha}$.

Для $\alpha$-коллекции $u \equiv\left(B_{a} \subset B \mid a \in A\right)_{\alpha}$ рассмотрим $\alpha$-классы

$$
\cup_{\alpha}\left(B_{a} \subset B \mid a \in A\right)_{\alpha} \equiv\left\{y \in \alpha \mid \exists x\left(x \in A \wedge y \in B_{x}\right)\right\}
$$


и

$$
\cap_{\alpha}\left(B_{a} \subset B \mid a \in A\right)_{\alpha} \equiv\left\{y \in \alpha \mid \forall x\left(x \in A \Longrightarrow y \in B_{x}\right)\right\}
$$

Ясно, что

$$
\cup_{\alpha}\left(B_{a} \subset B \mid a \in A\right)_{\alpha}=\operatorname{rng}_{\alpha} u \text {. }
$$

Следующая аксиома постулирует, что если $\alpha-$ универсальньй класс и $\left(B_{a} \subset B\right.$ $a \in A)_{\alpha}-\alpha$-коллекция $\alpha$-множеств $B_{a}$ из $\alpha$-класса $B$, индексированная $\alpha$-множеством $A$, то ее $\alpha$-обвединение

$$
\cup_{\alpha}\left(B_{a} \subset B \mid a \in A\right)_{\alpha}
$$

является $\alpha$-множеством.

А11 (Аксиома общего объединения). Вьполнено

$$
\begin{gathered}
\forall U\left(U \bowtie \Longrightarrow \forall X \forall Y \forall z \left(\left(X \in U \wedge Y \subset U \wedge\left(z \subset X *_{U} Y\right)\right.\right.\right. \\
\left.\left.\wedge \forall x(x \in X \Longrightarrow z\langle x\rangle \in U) \Longrightarrow\left(\operatorname{rng}_{U} z \in U\right)\right)\right) .
\end{gathered}
$$

В форме коллекции эта аксиома записывается следующим образом:

A11' (Аксиома общего объединения). Выполнено

$$
\begin{gathered}
\forall U\left(U \bowtie \Longrightarrow \forall X \forall Y \forall z \left(\left(X \in U \wedge Y \subset U \wedge\left(z \rightleftharpoons\left(Y_{a} \subset Y \mid a \in X\right)_{U}\right)\right.\right.\right. \\
\left.\left.\left.\wedge \forall x\left(x \in X \Longrightarrow Y_{x} \in U\right)\right) \Longrightarrow\left(\cup_{U}\left(Y_{a} \subset Y \mid a \in X\right)_{U} \in U\right)\right)\right) .
\end{gathered}
$$

Следующая аксиома служит для исключения самопринадлежности в универсальном классе $\alpha$.

А12 (Аксиома регулярности). Выполнено

$$
\forall U\left(U \bowtie \Longrightarrow \forall X\left(X \subset U \wedge X \neq \varnothing \Longrightarrow \exists x\left(x \in X \wedge x \cap_{U} X=\varnothing\right)\right)\right) .
$$

Следующая аксиома постулирует существование бесконечного множества.

А13 (Аксиома инфра-бесконечности). Вьполнено

$$
\exists X\left(X \in \mathfrak{a} \wedge \varnothing \in X \wedge \forall x\left(x \in X \Longrightarrow\left(x \cup_{\mathfrak{a}}\{x\}_{\mathfrak{a}} \in X\right)\right)\right) .
$$

Последняя аксиома постулирует существование функции выбора в любом универсальном классе $\alpha$.

А14 (Аксиома выбора). Вьполнено

$$
\begin{gathered}
\forall U\left(U \bowtie \Longrightarrow \forall X \left(X \in U \wedge X \neq \varnothing \Longrightarrow \exists z\left(\left(z \rightleftharpoons \mathscr{P}_{U}(X) \backslash\{\varnothing\}_{U} \rightarrow_{U} X\right)\right.\right.\right. \\
\left.\left.\left.\wedge \forall Y\left(Y \in \mathscr{P}_{U}(X) \backslash\{\varnothing\}_{U} \Longrightarrow z(Y) \in Y\right)\right)\right)\right) .
\end{gathered}
$$

Описание математических аксиом и аксиомных схем локальной теории множсеств $(L T S)$ закончено. Из них следует, что для каждого универсального класса $\alpha$ (в том числе и для инфра-универсума $\mathfrak{a})$ совокупность всех $\alpha$-классов и всех $\alpha$-множеств удовлетворяет всем аксиомам $N B G$ (см. [3]). Поэтому $\alpha$ является обычным $N B G$-универсумом. Это значит, что данная теория включает в себя $N B G$ и, следовательно, $Z F$. Если отбросить в AS2 условие $\alpha$-предикативности формулы $\varphi$, то в этом случае теория будет включать в себя теорию Мостовского-Келли-Морса $(M K M)$ (см. [2, добавление], [19, п. 5.6]).

Рассмотрим в LTS аксиому $\omega$-универсальности

$$
U(\omega)_{L T S} \equiv \exists X(\forall U \in X(U \bowtie) \wedge X \neq \varnothing \wedge \forall V \in X \exists W \in X(V \in W)),
$$

утверждающую существование бесконечного класса универсальных классов.

Следующая аксиома является отрицанием аксиомы $\omega$-универсальности. 
А15 (Аксиома экономности). Вьполнено $\neg U(\omega)_{L T S}$.

Локальную теорию множеств вместе с аксиомой А15 назовем локально-минимальной теорией множеств.

3. Некоторые начальные конструкции локальной теории множеств. Наша задача теперь - показать, что вся наивная теория категорий может быть адекватно формализована в рамках локальной теории множеств (с предикативной формой аксиомной схемы AS2).

Для этого нам сначала нужно ввести аналог координатной $\alpha$-пары $\langle A, B\rangle_{\alpha}$, который может работать также и для собственных $\alpha$-классов $A$ и $B$ (см. следствие к лемме 2 ).

А для этого нужно сначала ввести натуральные числа.

Обозначим a-множество, существование которого постулируется аксиомой инфрабесконечности, через $\pi$.

ЛЕмма 3. Пусть $\alpha-$ универсальный класс. Тогда $\varnothing \in \alpha$.

ДокАЗАТЕЛЬСтво. По А13 $\pi \in \mathfrak{a}$ и $\varnothing \in \pi$. Тогда по А7 $\pi \subset \mathfrak{a}$ и, следовательно, $\varnothing \in \mathfrak{a}$. Отсюда в силу А5 $\varnothing \in \alpha$.

Рассмотрим а-класс

$$
\varkappa \equiv\left\{Y \in \mathfrak{a} \mid Y \subset \pi \wedge \varnothing \in Y \wedge \forall y a\left(y \in \mathfrak{a} \wedge y \in Y \Longrightarrow\left(y \cup_{\mathfrak{a}}\{y\}_{\mathfrak{a}} \in Y\right)\right)\right\} .
$$

Так как по AS2 $\varkappa \subset \mathscr{P}_{\mathfrak{a}}(\pi)$, то из А9 и А8 следует, что $\varkappa$ является $\mathfrak{a}$-множеством.

Рассмотрим а-класс

$$
\omega \equiv\{y \in \mathfrak{a} \mid \forall Y(Y \in \mathfrak{a} \wedge Y \in \varkappa \Longrightarrow y \in Y)\}
$$

Лемма 4. Пусть $\alpha-$ универсальный класс. Тогда $\omega \in \alpha$.

ДокАЗАтЕЛьСтво. По А13 и AS2 следует, что $\pi \in \varkappa$. Если $y \in \omega$, то AS2 и $\pi \in \varkappa$ влекут $y \in \pi$. Значит, $\omega \subset \pi$. По А $8 \omega \in \mathfrak{a}$. Отсюда в силу А5 $\omega \in \alpha$.

В силу этой леммы мы можем назвать класс $\omega$ “множеством" натуральных чисел, поскольку $\omega$ является множеством в любом универсальном классе.

Рассмотрим начальные натуральные числа

$$
0 \equiv \varnothing, \quad 1 \equiv 0 \cup_{\mathfrak{a}}\{0\}_{\mathfrak{a}}, \quad 2 \equiv 1 \cup_{\mathfrak{a}}\{1\}_{\mathfrak{a}}, \quad 3 \equiv 2 \cup_{\mathfrak{a}}\{2\}_{\mathfrak{a}}, \quad \ldots
$$

Из определения $\varkappa$ и $\omega$ следует, что $0,1,2,3, \ldots \in \omega$. Из леммы 4 и А7 следует, что $0,1,2,3, \ldots \in \alpha$ для каждого универсального класса $\alpha$.

Последнее утверждение обеспечивает корректность следующих определений.

Пусть $\alpha$ - фиксированньй универсальный класс. Пусть даны $\alpha$-классы $A, A^{\prime}, A^{\prime \prime}, \ldots$, где штрих использован только ради единообразия обозначений.

$\alpha$-коллекцию $\left(A_{i} \subset \alpha \mid i \in 2\right)_{\alpha}$ такую, что $A_{0} \equiv A$ и $A_{1} \equiv A^{\prime}$, назовем (многозначной) последовательной $\alpha$-парой $\alpha$-классов $A$ и $A^{\prime}$ и обозначим через $\left(A, A^{\prime}\right)_{\alpha}$; $\alpha$-коллекцию $\left(A_{i} \subset \alpha \mid i \in 3\right)_{\alpha}$ такую, что $A_{0}=A, A_{1}=A^{\prime}$ и $A_{2}=A^{\prime \prime}$, назовем (многозначной) последовательной $\alpha$-тройкой $\alpha$-классов $A, A^{\prime} u A^{\prime \prime}$ и обозначим через $\left(A, A^{\prime}, A^{\prime \prime}\right)_{\alpha}$, и т.д.

Пусть теперь даны $\alpha$-множества $a, a^{\prime}, a^{\prime \prime}, \ldots$

Простую $\alpha$-коллекцию $\left(a_{i} \in \alpha \mid i \in 2\right) \alpha$ такую, что $a_{0}=a$ и $a_{1}=a^{\prime}$, назовем простой последовательной $\alpha$-парой $\alpha$-множеств а и $a^{\prime}$ и обозначим через $\left(a, a^{\prime}\right)_{\alpha}$. Простую $\alpha$-коллекцию $\left(a_{i} \in \alpha \mid i \in 3\right)_{\alpha}$ такую, что $a_{0}=a, a_{1}=a^{\prime}$ и $a_{2}=a^{\prime \prime}$, назовем простой последовательной $\alpha$-тройкой $\alpha$-множеств $a, a^{\prime} u a^{\prime \prime}$ и обозначим через $\left(a, a^{\prime}, a^{\prime \prime}\right)_{\alpha}$, и т.д. 
Лемма 5. Пусть $\alpha$-универсальный класс, $A, A^{\prime}, B, B^{\prime}-\alpha$-классы $и a, a^{\prime}, b$, $b^{\prime}-\alpha$-множества. Тогда

1) $е с л u\left(A, A^{\prime}\right)_{\alpha}=\left(B, B^{\prime}\right)_{\alpha}$, mo $A=B u A^{\prime}=B^{\prime}$;

2) если $\left(a, a^{\prime}\right)_{\alpha}=\left(b, b^{\prime}\right)_{\alpha}$, mo $a=a^{\prime} u b=b^{\prime}$.

ДоказАТЕльство. 1) Из определения $\alpha$-классов $u \equiv\left(A, A^{\prime}\right)_{\alpha}$ и $v \equiv\left(B, B^{\prime}\right)_{\alpha}$ следует, что $u \subset 2 *_{\alpha} \alpha, v \subset 2 *_{\alpha} \alpha, u\langle 0\rangle=A, u\langle 1\rangle=A^{\prime}, v\langle 0\rangle=B$ и $v\langle 1\rangle=B^{\prime}$. Если $x \in A=u\langle 0\rangle$, топо AS2 $x \in \alpha \wedge\langle 0, x\rangle_{\alpha} \in u$. Так как $u=v$, то $x \in \alpha \wedge\langle 0, x\rangle_{\alpha} \in v$. Снова по AS2 $x \in v\langle 0\rangle=B$. Значит, $A \subset B$. Обратное вложение проверяется аналогично. Таким образом, $A=B$. Второе равенство доказьвается аналогично.

2) Из определения $\alpha$-классов $u \equiv\left(a, a^{\prime}\right)_{\alpha}$ и $v \equiv\left(b, b^{\prime}\right)_{\alpha}$ следует, что $u \rightleftharpoons 2 \rightarrow_{\alpha} \alpha$, $v \rightleftharpoons 2 \rightarrow_{\alpha} \alpha, u(0)=a, u(1)=a^{\prime}, v(0)=b$ и $v(1)=b^{\prime}$. По условию $a=u(0) \in u\langle 0\rangle$. Поэтому по AS2 $a \in \alpha \wedge\langle 0, a\rangle_{\alpha} \in u$. Так как $u=v$, то $a \in \alpha \wedge\langle 0, a\rangle_{\alpha} \in v$. Отсюда по AS2 $a \in v\langle 0\rangle=\{v(0)\}_{\alpha}$. Снова применяя AS2, получаем $a=v(0)=b$. Аналогично проверяется, что $a^{\prime}=b^{\prime}$.

Из леммы 5 следует, что $\alpha$-пары $\left(A, A^{\prime}\right)_{\alpha}$ и $\left(a, a^{\prime}\right)_{\alpha}$ обладают свойством $\alpha$-пары Куратовского $\left\langle a, a^{\prime}\right\rangle_{\alpha}$ из следствия к лемме 2. Однако в отличие от последней $\alpha$-пара $\left(A, A^{\prime}\right)_{\alpha}$ работает также и для собственных $\alpha$-классов.

Используя понятия коллекции $\alpha$-классов и конечного набора $\alpha$-классов, определим теперь понятия соответствующих произведений. Пусть дана некоторая $\alpha$-коллекция $u \equiv\left(A_{i} \subset \alpha \mid i \in I\right)_{\alpha}$, индексированная непустым $\alpha$-классом $I$.

$\alpha$-класс

$$
\prod_{\alpha}\left(A_{i} \subset \alpha \mid i \in I\right)_{\alpha} \equiv\left\{z \in \alpha \mid\left(z \rightleftharpoons I \rightarrow_{\alpha} \alpha\right) \wedge \forall x\left(x \in \alpha \wedge x \in I \Longrightarrow z(x) \in A_{x}\right)\right\}
$$

назовем $\alpha$-произведением $\alpha$-коллекиии $u$. Если все $\alpha$-классы $A_{i}$ не пусты, то из А14 следует, что $\alpha$-произведение тоже не пусто.

В частном случае для $\alpha$-классов $A, A^{\prime}, A^{\prime \prime}, \ldots \alpha$-классы $\prod_{\alpha}\left(A, A^{\prime}\right)_{\alpha}, \prod_{\alpha}\left(A, A^{\prime}\right.$, $\left.A^{\prime \prime}\right)_{\alpha}, \ldots$ назовем $\alpha$-произведением $\alpha$-пары $\left(A, A^{\prime}\right)_{\alpha}, \alpha$-тройки $\left(A, A^{\prime}, A^{\prime \prime}\right)_{\alpha}, \ldots$ и обозначим через $A \times{ }_{\alpha} A^{\prime}, A \times{ }_{\alpha} A^{\prime} \times{ }_{\alpha} A^{\prime \prime}, \ldots$

Лемма 6. Пусть $\alpha-$ универсальный класс и $A, A^{\prime}-\alpha$-классы. Тогда

$$
A \times_{\alpha} A^{\prime}=\left\{x \in \alpha \mid \exists y \exists y^{\prime}\left(y \in \alpha \wedge y \in A \wedge y^{\prime} \in \alpha \wedge y^{\prime} \in A^{\prime} \wedge x=\left(y, y^{\prime}\right)_{\alpha}\right)\right\}
$$

ДокАЗАтЕЛЬСтво. Обозначим левую часть этого равенства через $P$, а правую через $Q$. По определению $\left(A, A^{\prime}\right)_{\alpha} \equiv\left(A_{i} \subset \alpha \mid i \in 2\right)_{\alpha}$, где $A_{0}=A$ и $A_{1}=A^{\prime}$. Поэтому

$$
P=\left\{z \in \alpha \mid\left(z \rightleftharpoons 2 \rightarrow_{\alpha} \alpha\right) \wedge \forall x\left(x \in \alpha \wedge x \in 2 \Longrightarrow z(x) \in A_{x}\right)\right\} .
$$

Пусть $p \in P$. Тогда по AS2 $p \rightleftharpoons 2 \rightarrow_{\alpha} \alpha, p(0) \in A_{0}$ и $p(1) \in A_{1}$. Обозначим $p(0)$ через $a$ и $a_{0}$; соответственно $p(1)$ обозначим через $a^{\prime}$ и $a_{1}$. Тогда $p \rightleftharpoons\left(a_{i} \in \alpha \mid i \in 2\right)_{\alpha}$. Рассмотрим $\alpha$-пару $\left(a, a^{\prime}\right)_{\alpha}$. По определению $\left(a, a^{\prime}\right)_{\alpha} \equiv\left(x_{i} \in \alpha \mid i \in 2\right)_{\alpha}$, где $x_{0}=a$ и $x_{1}=a^{\prime}$. Так как $x_{0}=a$ и $x_{1}=a_{1}$, то $\left(a, a^{\prime}\right)_{\alpha}=\left(a_{i} \in \alpha \mid i \in 2\right)$. Следовательно, $\left(a, a^{\prime}\right)_{\alpha}=p$. Так как $a \in A \subset \alpha$ и $a^{\prime} \in A^{\prime} \subset \alpha$, мы приходим к $\alpha$-предикативной формуле

$$
\exists y \exists y^{\prime}\left(y \in \alpha \wedge y \in A \wedge y^{\prime} \in \alpha \wedge y^{\prime} \in A^{\prime} \wedge p=\left(y, y^{\prime}\right)_{\alpha}\right)
$$


Используя AS2, мы получаем $p \in Q$. Значит, $P \subset Q$.

Обратно, пусть $q \in Q$. Тогда по AS2 $q=\left(b, b^{\prime}\right)_{\alpha}$ для некоторых $b \in A$ и $b^{\prime} \in A^{\prime}$. По определению $\left(b, b^{\prime}\right)_{\alpha} \equiv\left(y_{i} \in \alpha \mid i \in 2\right)_{\alpha}$, где $y_{0}=b$ и $y_{1}=b^{\prime}$. Следовательно, $q \rightleftharpoons 2 \rightarrow_{\alpha} \alpha, q(0)=y_{0}=b \in A$ и $q(1)=y_{1}=b^{\prime} \in A^{\prime}$. Используя AS2, мы получаем $q \in P$. Значит, $Q \subset P$.

Из этой леммы следует, что $\alpha$-произведение $A \times{ }_{\alpha} A^{\prime}$ сходно с координатным $\alpha$-произведением $A *_{\alpha} A^{\prime}$, но в отличие от последнего является частным случаем общего произведения $\prod_{\alpha}\left(A_{i} \subset \alpha \mid i \in I\right)_{\alpha}$.

4. Формализация наивной теории категорий в рамках локальной теории множеств. Под наивным понятием категории понимается понятие метакатегории, данное Маклейном в [14]. Согласно [14] метакатегория состоит из обвектов $\pi, \varkappa, \rho, \ldots$, стрелок $F, G, H, \ldots$ и четырех операций

$$
F \mapsto \operatorname{dom} F, \quad F \mapsto \operatorname{codom} F, \quad \pi \mapsto i_{\pi}, \quad(F, G) \mapsto G \circ F
$$

удовлетворяющих некоторым дополнительным условиям. К сожалению, под это определение подходит даже такой патологический объект, как метакатегория всех метакатегорий.

В связи с внутренней противоречивостью понятия метакатегории и возникла проблема формализации наивной теории категорий. Цель такой формализации состоит в том, чтобы в некоторой точно описанной аксиоматике (теоретико-множественной, стрелочной или смешанной) дать точное определение некоторого объекта, которое

1) соответствует наивному представлению о категории;

2) является замкнутым относительно всех операций и конструкций наивной теории категорий;

3) включает в себя все известные содержательные конкретныепримеры категорий;

4) отсекает такие наивные патологические примеры, как метакатегория всех метакатегорий.

Используя понятия предыдущих пунктов, мы можем формализовать наивное понятие категории следуюшим образом.

Для каж дого универсального класса $\alpha$ мы определим $\alpha$-категорию как большую двусортную алгебраическую систему с двумя отношениями и одной операцией. Для этой цели будем использовать понятия $\alpha$-коллекции $\alpha$-классов и $\alpha$-пары $\alpha$-классов, введенные ранее.

Рассмотрим символьное $\mathfrak{a}$-множество $\Omega_{c}$, состояшее из трех $\mathfrak{a}$-множеств, обозначенных знаками \#, о и $\leftrightarrow$. Эти знаки будем назьвать знаком разбиения, знаком композиции и знаком идентификаиии соответственно. Множество $\Omega_{c}$ назовем сигнатурой категории. Это понятие аналогично таким понятиям, как сигнатура абелевой группь $\Omega_{g} \equiv\{\{0\},-,+\}$, сигнатура кольиа $\Omega_{r} \equiv\{\{0\},-,+, \cdot\}$ и т.п.

Так как по А5 a $\subset \alpha$, то $\Omega_{c}$ является также $\alpha$-множеством для каждого универсального класса $\alpha$.

Зафиксируем некоторый универсальньй класс $\alpha$.

Рассмотрим $\alpha$-пару $A \equiv(\mathrm{Obj}, \mathrm{Arr})_{\alpha}$, состоящую из некоторых $\alpha$-классов Obj и Arr. Рассмотрим также $\alpha$-коллекцию

$$
s_{c} \equiv\left(A_{\omega} \subset \alpha \mid \omega \in \Omega_{c}\right)_{\alpha}
$$


с тремя компонентами $A_{\#}, A_{\circ}$ и $A_{\leftrightarrow}$. Далее для удобства эти компоненты будем обозначать в более привычном перевернутом виде $\# A,{ }^{\circ} A$ и $\leftrightarrow A_{A}$, означающем разбиение $\boldsymbol{\theta}$ $A$, композииию в $A$ и идентификацию в $A$. Это обозначение удобно потому, что при выборе другой $\alpha$-пары $B$ мы будем иметь \# $B$, о $_{B}$ и $\leftrightarrow_{B}$.

Рассмотрим $\alpha$-класс $\mathscr{C} \equiv\left(A, s_{c}\right)$. Классы $A$ и $s_{c}$ будем назьвать соответственно носителем и структурой класса $\mathscr{C}$. Классы Obj и Arr будем называть соответственно классом облектов и классом стрелок класса $\mathscr{C}$.

Дадим теперь определение $\alpha$-категории. Мы вьнуждены привести точное формальное определение по той причине, что оно будет использовано далее в статье. Для того, чтобы сделать его более понятным, мы разобьем его на отдельные части и снабдим каждую часть параллельным комментарием с использованием стандартных обозначений и понятий.

$\alpha$-класс $\mathscr{C}$ назовем $\alpha$-категорией, если

$$
\operatorname{cat}_{\alpha}(\mathscr{C}) \equiv \operatorname{cat}_{\alpha}(X \| \mathscr{C})
$$

где аксиома $\alpha$-категории $\operatorname{cat}_{\alpha}(X)$ со свободной переменной $X$ имеет следующий вид:

$$
\begin{aligned}
\operatorname{cat}_{\alpha}(X) \equiv & (X \subset \alpha) \wedge \exists Y^{\prime}, Y^{\prime \prime}, Z\left(Y^{\prime}, Y^{\prime \prime}, Z \subset \alpha \wedge X=\left(\left(Y^{\prime}, Y^{\prime \prime}\right)_{\alpha}, Z\right)_{\alpha}\right. \\
& \left.\wedge\left(Z \rightleftharpoons \Omega_{c} \longrightarrow_{\alpha} \alpha\right) \wedge A_{c} 1\left(Y^{\prime}, Y^{\prime \prime}, Z\right) \wedge \cdots \wedge A_{c} 7\left(Y^{\prime}, Y^{\prime \prime}, Z\right)\right),
\end{aligned}
$$

где формулы $A_{c} 1-A_{c} 7$ приводятся ниже.

$\left(\right.$ Свойство $\operatorname{cat}_{\alpha}(\mathscr{C})$ означает, что $\mathscr{C} \subset \alpha, \mathscr{C}=\left(A, s_{c}\right)_{\alpha}, A=(\mathrm{Obj}, \mathrm{Arr})_{\alpha}, s_{c}=$ $\left(\omega_{A} \subset \alpha \mid \omega \in \Omega\right)_{\alpha}$, а $\alpha$-классы Obj, Arr, \# $\#_{A} \equiv s_{c}\langle \#\rangle, \circ_{A} \equiv s_{c}\left\langle\right.$ о и $\leftrightarrow_{A} \equiv s_{c}\langle\leftrightarrow\rangle$ обладают приводимыми ниже свойствами $P_{c} 1-P_{c} 7$.)

Формула $A_{c} 1$ :

$$
A_{c} 1 \equiv\left(Z\langle \#\rangle \subset\left(Y^{\prime} \times_{\alpha} Y^{\prime}\right) *_{\alpha} Y^{\prime \prime}\right) \wedge\left(Z\langle\circ\rangle \subset\left(Y^{\prime \prime} \times_{\alpha} Y^{\prime \prime}\right) *_{\alpha} Y^{\prime \prime}\right) \wedge\left(Z\langle\leftrightarrow\rangle \subset Y^{\prime} *_{\alpha} Y^{\prime \prime}\right) .
$$

(Свойство $P_{c} 1 \equiv A_{c} 1\left(\mathrm{Obj}, \mathrm{Arr}, s_{c}\right)$ означает, что компоненты $\#_{A}$, о $_{A}$ и $\leftrightarrow_{A}$ структуры $s_{c}$ являются двусортными алгебраическими $\alpha$-соответствиями.)

Формула $A_{c} 2$ :

$$
\begin{gathered}
A_{c} 2 \equiv\left(\operatorname{rng}_{\alpha} Z\langle \#\rangle=Y^{\prime \prime}\right) \wedge\left(\forall x, y \in \alpha\left(x, y \in Y^{\prime} \times_{\alpha} Y^{\prime} \wedge x \neq y\right.\right. \\
\left.\left.\Longrightarrow Z\langle \#\rangle\langle x\rangle \cap_{\alpha} Z\langle \#\rangle\langle y\rangle=\varnothing\right)\right)
\end{gathered}
$$

(Свойство $P_{c} 2 \equiv A_{c} 2\left(\mathrm{Obj}, \operatorname{Arr}, s_{c}\right)$ означает, что $\alpha$-класс стрелок Arr является объединением $\alpha$-коллекции

$$
\left(\operatorname{Arr}(\pi, \varkappa) \subset \operatorname{Arr} \mid(\pi, \varkappa)_{\alpha} \in \operatorname{Obj} \times{ }_{\alpha} \mathrm{Obj}\right)_{\alpha}
$$

попарно непересекающихся $\alpha$-подклассов $\operatorname{Arr}(\pi, \varkappa)$, состояших из стрелок $F: \pi \dot{\rightarrow} \varkappa$ из объекта $\pi$ в облект $\varkappa$ для всех $\alpha$-пар $(\pi, \varkappa)_{\alpha}$ объектов $\left.\pi, \varkappa \in \mathrm{Obj.}\right)$

Формула $A_{c} 3$ :

$$
\begin{aligned}
A_{c} 3 \equiv & \left(\operatorname{dom}_{\alpha} Z\langle\circ\rangle=\left\{x \in \alpha \mid \exists x^{\prime}, y^{\prime}, z^{\prime}, y^{\prime \prime}, z^{\prime \prime} \in \alpha\left(x^{\prime}, y^{\prime}, z^{\prime} \in Y^{\prime}\right.\right.\right. \\
& \left.\wedge y^{\prime \prime}, z^{\prime \prime} \in Y^{\prime \prime} \wedge\left(y^{\prime \prime} \in Z\langle \#\rangle\left\langle\left(x^{\prime}, y^{\prime}\right)_{\alpha}\right\rangle\right) \wedge z^{\prime \prime} \in Z\langle \#\rangle\left\langle\left(y^{\prime}, z^{\prime}\right)_{\alpha}\right\rangle\right) \\
& \left.\left.\wedge\left(x=\left(y^{\prime \prime}, z^{\prime \prime}\right)_{\alpha}\right)\right\}\right) \wedge\left(Z\langle\circ\rangle \rightleftharpoons \operatorname{dom}_{\alpha} Z\langle\circ\rangle \rightarrow_{\alpha} Y^{\prime \prime}\right) .
\end{aligned}
$$


(Свойство $P_{c} 3 \equiv A_{c} 3\left(\mathrm{Obj}, \mathrm{Arr}, s_{c}\right)$ означает, что композиция о $A$ является частично определенной бинарной операцией и стрелка $G \circ F \equiv \circ_{A}(F, G)$ определена только для стрелок вида $F: \pi \dot{\rightarrow} \varkappa$ и $G: \varkappa \dot{\rightarrow} \rho$.)

Формула $A_{c} 4$ :

$$
A_{c} 4 \equiv\left(Z\langle\leftrightarrow\rangle \rightleftharpoons\left(Y^{\prime} \rightarrow_{\alpha} Y^{\prime \prime}\right) \wedge \forall x^{\prime} \in \alpha\left(x^{\prime} \in Y^{\prime} \Longrightarrow\left(Z\langle\leftrightarrow\rangle\left(x^{\prime}\right) \in Z\langle \#\rangle\left\langle\left(x^{\prime}, x^{\prime}\right)_{\alpha}\right\rangle\right)\right)\right) .
$$

(Свойство $P_{c} 4 \equiv A_{c} 4\left(\mathrm{Obj}, \mathrm{Arr}, s_{c}\right)$ означает, что идентификация $\leftrightarrow_{A}$ является $\alpha$-отображением из Obj в $\mathrm{Arr}$, сопоставляющим каждому объекту $\pi$ некоторую стрелку $i_{\pi} \equiv \leftrightarrow_{A}(\pi)$ из $\pi$ в $\pi$.)

Формула $A_{c} 5$ :

$$
\begin{aligned}
A_{c} 5 \equiv & \forall x^{\prime}, y^{\prime}, z^{\prime} \in \alpha\left(x^{\prime}, y^{\prime}, z^{\prime} \in Y^{\prime}\right. \\
& \left.\Longrightarrow Z\langle 0\rangle\left[Z\langle \#\rangle\left\langle\left(x^{\prime}, y^{\prime}\right)_{\alpha}\right\rangle \times_{\alpha} Z\langle \#\rangle\left\langle\left(y^{\prime}, z^{\prime}\right)_{\alpha}\right\rangle\right] \subset Z\langle \#\rangle\left\langle\left(x^{\prime}, z^{\prime}\right)_{\alpha}\right\rangle\right) .
\end{aligned}
$$

(Свойство $P_{c} 5 \equiv A_{c} 5\left(\mathrm{Obj}, \mathrm{Arr}, s_{c}\right)$ означает, что для стрелок $F: \pi \dot{\rightarrow} \varkappa$ и $G: \varkappa \dot{\rightarrow} \rho$ их композиция дает стрелку $G \circ F: \pi \dot{\rightarrow} \rho$.)

Формула $A_{c} 6$ :

$$
\begin{aligned}
A_{c} 6 \equiv & \forall x^{\prime}, y^{\prime}, z^{\prime}, u^{\prime}, x^{\prime \prime}, y^{\prime \prime}, z^{\prime \prime} \in \alpha\left(x^{\prime}, y^{\prime}, z^{\prime}, u^{\prime} \in Y^{\prime} \wedge x^{\prime \prime}, y^{\prime \prime}, z^{\prime \prime} \in Y^{\prime \prime}\right. \\
& \wedge\left(x^{\prime \prime} \in Z\langle \#\rangle\left\langle\left(x^{\prime}, y^{\prime}\right)_{\alpha}\right\rangle\right) \wedge\left(y^{\prime \prime} \in Z\langle \#\rangle\left\langle\left(y^{\prime}, z^{\prime}\right)_{\alpha}\right\rangle\right) \wedge\left(z^{\prime \prime} \in Z\langle \#\rangle\left\langle\left(z^{\prime}, u^{\prime}\right)_{\alpha}\right\rangle\right) \\
& \left.\Longrightarrow\left(Z\langle\circ\rangle\left(\left(Z\langle\circ\rangle\left(\left(x^{\prime \prime}, y^{\prime \prime}\right)_{\alpha}\right), z^{\prime \prime}\right)_{\alpha}\right)=Z\langle\circ\rangle\left(\left(x^{\prime \prime}, Z\langle\circ\rangle\left(\left(y^{\prime \prime}, z^{\prime \prime}\right)_{\alpha}\right)\right)_{\alpha}\right)\right)\right) .
\end{aligned}
$$

(Свойство $P_{c} 6 \equiv A_{c} 6\left(\mathrm{Obj}, \mathrm{Arr}, s_{c}\right)$ выражает свойство ассоииативности $H \circ(G \circ F)$

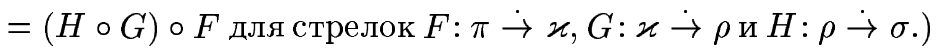

Формула $A_{c} 7$ :

$$
\begin{aligned}
A_{c} 7 \equiv & \forall x^{\prime}, y^{\prime}, z^{\prime}, x^{\prime \prime}, y^{\prime \prime} \in \alpha\left(x^{\prime}, y^{\prime}, z^{\prime} \in Y^{\prime} \wedge x^{\prime \prime}, y^{\prime \prime}, \in Y^{\prime \prime}\right. \\
& \wedge\left(x^{\prime \prime} \in Z\langle \#\rangle\left\langle\left(y^{\prime}, x^{\prime}\right)_{\alpha}\right\rangle\right) \wedge\left(y^{\prime \prime} \in Z\langle \#\rangle\left\langle\left(x^{\prime}, z^{\prime}\right)_{\alpha}\right\rangle\right) \\
& \left.\Longrightarrow\left(\left(Z\langle\circ\rangle\left(\left(x^{\prime \prime}, Z\langle\leftrightarrow\rangle\left(x^{\prime \prime}\right)\right)_{\alpha}\right)=x^{\prime \prime}\right) \wedge\left(Z\langle\circ\rangle\left(\left(Z\langle\leftrightarrow\rangle\left(x^{\prime}\right), y^{\prime \prime}\right)_{\alpha}\right)=y^{\prime \prime}\right)\right)\right) .
\end{aligned}
$$

(Свойство $P_{c} 7 \equiv A_{c} 7\left(\mathrm{Obj}, \mathrm{Arr}, s_{c}\right)$ выражает свойство тождественности стрел$\kappa и i_{\pi}: \pi \rightarrow \pi$ в том смысле, что $i_{\pi} \circ F=F$ и $G \circ i_{\pi}=G$ для стрелок $F: \varkappa \dot{\rightarrow} \pi$ и $G: \pi \rightarrow \rho$.

$\alpha$-категория $\mathscr{C}$ назьвается малой, если $\mathscr{C}$ является $\alpha$-множеством. В противном случае она называется больиой.

Далее определения будем давать в более свободной форме. При необходимости они могут быть записаны в таком же строгом виде, как и определение $\alpha$-категории.

Формализуем теперь наивное понятие функтора. Пусть даны $\alpha$-категории

$$
\mathscr{C} \equiv\left((\operatorname{Obj} \mathscr{C}, \operatorname{Arr} \mathscr{C})_{\alpha}, s_{c}\right)_{\alpha} \quad \text { и } \quad \mathscr{D} \equiv\left((\operatorname{Obj} \mathscr{D}, \operatorname{Arr} \mathscr{D})_{\alpha}, t_{c}\right)_{\alpha}
$$

$\alpha$-класс $\Phi \equiv\left(\phi, \phi_{a}\right)_{\alpha}$ назовем (ковариантным $\alpha$-функтором из $\alpha$-категории $\mathscr{C}$ в $\alpha$-категорию $\mathscr{D}$, если $\alpha$-классы $\phi$ и $\phi_{a}$ входят в следующие формулы (записанные неформальным образом):

- формула $P_{f} 1: \phi \rightleftharpoons \operatorname{Obj} \mathscr{C} \rightarrow{ }_{\alpha} \operatorname{Obj} \mathscr{D} ;$

- формула $P_{f} 2: \phi_{a} \rightleftharpoons \operatorname{Arr} \mathscr{C} \rightarrow_{\alpha} \operatorname{Arr} \mathscr{D}$; 
- формула $P_{f} 3: \phi_{a}(F) \in \operatorname{Arr}_{\mathscr{D}}(\phi(\pi), \phi(\varkappa))$ для всех объектов $\pi, \varkappa \in \operatorname{Obj} \mathscr{C}$ и каждой стрелки $F \in \operatorname{Arr}_{\mathscr{C}}(\pi, \varkappa)$;

- формула $P_{f} 4: \phi_{a}(G \circ F)=\phi_{a}(G) \circ \phi_{a}(F)$ для всех объектов $\pi, \varkappa, \rho \in \operatorname{Obj} \mathscr{C}$ и каждых стрелок $F \in \operatorname{Arr}_{\mathscr{C}}(\pi, \varkappa)$ и $G \in \operatorname{Arr}_{\mathscr{C}}(\varkappa, \rho) ;$

- формула $P_{f} 5: \phi_{a}\left(i_{\pi}\right)=i_{\phi(\pi)}$ для каждого объекта $\pi \in \operatorname{Obj} \mathscr{C}$.

$\alpha$-функторы являются в точности гомоморфизмами между $\alpha$-категориями как алгебраическими системами.

$\alpha$-функтор $\Phi$ из $\mathscr{C}$ в $\mathscr{D}$ будем обозначать через $\Phi: \mathscr{C} \stackrel{f}{\rightarrow} \alpha \mathscr{D}$. $\Phi$ ормулу, выражающую для $\alpha$-класса $\Phi$ свойство быть $\alpha$-функтором из $\alpha$-категории $\mathscr{C}$ в $\alpha$-категорию $\mathscr{D}$, будем обозначать через $\Phi \rightleftharpoons \mathscr{C} \stackrel{f}{\rightarrow}_{\alpha} \mathscr{D}$.

$\alpha$-тройку $F \equiv(\Phi, \mathscr{C}, \mathscr{D})_{\alpha}$ будем называть (функторной) стрелкой, определяемой $\alpha$-категориями $\mathscr{C}$ и $\mathscr{D}$ и $\alpha$-функтором $\Phi: \mathscr{C} \stackrel{f}{\rightarrow} \mathscr{D}$.

Композичия $\alpha$-функторов

$$
\Phi \equiv\left(\phi, \phi_{a}\right)_{\alpha}: \mathscr{C} \stackrel{f}{\rightarrow}_{\alpha} \mathscr{D} \quad \text { и } \quad \Psi \equiv\left(\psi, \psi_{a}\right)_{\alpha}: \mathscr{D} \stackrel{f}{\rightarrow}_{\alpha} \mathscr{E}
$$

есть $\alpha$-фрунктор

$$
\Psi \circ \Phi \equiv\left(\psi \circ \phi, \psi_{a} \circ \phi_{a}\right)_{\alpha}: \mathscr{C} \stackrel{f}{\rightarrow}_{\alpha} \mathscr{E}
$$

Эта композиция задает композицию стрелок $F \equiv(\Phi, \mathscr{C}, \mathscr{D})_{\alpha}$ и $G \equiv(\Psi, \mathscr{D}, \mathscr{E})_{\alpha}$ как стрелку $G \circ F \equiv(\Psi \circ \Phi, \mathscr{C}, \mathscr{E})_{\alpha}$.

Тождественный $\alpha$-функтор для $\alpha$-категории $\mathscr{C}$ есть $\alpha$-функтор $I_{\mathscr{C}} \equiv\left(\operatorname{id}_{\mathrm{Obj}} \mathscr{C}\right.$, $\left.\operatorname{id}_{\mathrm{Arr}} \mathscr{C}\right)_{\alpha}$, состояший из двух тождественньх отображений для $\alpha$-классов Obj $\mathscr{C}$ и $\operatorname{Arr} \mathscr{C}$ соответственно. Этот $\alpha$-функтор задает тождественную стрелку для $\alpha$-категории $\mathscr{C}$ как стрелку $i_{\mathscr{C}} \equiv\left(I_{\mathscr{C}}, \mathscr{C}, \mathscr{C}\right)_{\alpha}$.

Формализуем теперь наивное понятие естественного преобразования. Пусть даны фиксированные $\alpha$-категории $\mathscr{C}$ и $\mathscr{D}$ и два $\alpha$-функтора $\Phi: \mathscr{C} \stackrel{f}{\rightarrow}{ }_{\alpha} \mathscr{D}$ и $\Psi: \mathscr{C} \stackrel{f}{\rightarrow} \alpha \mathscr{D}$.

$\alpha$-класс

$$
T \equiv\left(t_{\pi} \in \operatorname{Arr} \mathscr{D} \mid \pi \in \operatorname{Obj} \mathscr{C}\right)_{\alpha}
$$

назовем (естественным $\alpha$-преобразованием из $\alpha$-функтора $\Phi \equiv\left(\phi, \phi_{a}\right)_{\alpha}$ в $\alpha$-функтор $\Psi \equiv\left(\psi, \psi_{a}\right)_{\alpha}$, если

1) $t_{\pi} \in \operatorname{Arr}_{\mathscr{D}}(\varphi(\pi), \psi(\pi))$ для каждого объекта $\pi$ из $\mathrm{Obj} \mathscr{C}$;

2) $\psi_{a}(F) \circ t_{\pi}=t_{\varkappa} \circ \phi_{a}(F)$ для каждьх объектов $\pi, \varkappa$ из Obj $\mathscr{C}$ и каждой стрелки $F$ из $\operatorname{Arr}_{\mathscr{C}}(\pi, \varkappa)$.

$\alpha$-преобразование из $\Phi$ в $\Psi$ будем обозначать через $T: \Phi \stackrel{t}{\rightarrow} \alpha \Psi$. ормулу, выражающую для $\alpha$-класса $T$ свойство быть $\alpha$-преобразованием из $\alpha$-функтора $\Phi$ в $\alpha$-функтор $\Psi$, будем обозначать через $T \rightleftharpoons \Phi \stackrel{t}{\rightarrow}_{\alpha} \Psi$.

$\alpha$-тройку $F \equiv(T, \Phi, \Psi)_{\alpha}$ будем называть (преобразовательной) стрелкой, определяемой $\alpha$-функторами $\Phi$ и $\Psi$ и $\alpha$-преобразованием $T: \Phi \stackrel{t}{\rightarrow} \Psi$.

Композичия $\alpha$-преобразований $T \equiv\left(t_{\pi} \in \operatorname{Arr} \mathscr{D} \mid \pi \in \operatorname{Obj}_{\mathscr{C}}\right)_{\alpha}: \Phi \stackrel{t}{\rightarrow}{ }_{\alpha} \Psi$ и $U \equiv$ $\left(u_{\pi} \in \operatorname{Arr} \mathscr{D} \mid \pi \in \operatorname{Obj}_{\mathscr{C}}\right)_{\alpha}: \Psi \stackrel{t}{\rightarrow} \alpha \Omega$ есть $\alpha$-преобразование $U \circ T \equiv\left(u_{\pi} \circ t_{\pi} \in \operatorname{Arr} \mathscr{D} \mid\right.$ $\left.\pi \in \operatorname{Obj}^{C}\right)_{\alpha}: \Phi \stackrel{t}{\rightarrow}{ }_{\alpha} \Omega$. Эта композищия задает композииию стрелок $F \equiv(T, \Phi, \Psi)_{\alpha}$ и $G \equiv(U, \Psi, \Omega)_{\alpha}$ как стрелку $G \circ F \equiv(U \circ T, \Phi, \Omega)_{\alpha}$. 
Тождественное $\alpha$-преобразование для $\alpha$-функтора $\Phi: \mathscr{C} \stackrel{f}{\rightarrow}_{\alpha} \mathscr{D}$ есть $\alpha$-преобразование

$$
I_{\Phi} \equiv\left(i_{\phi(\pi)} \in \operatorname{Arr} \mathscr{D} \mid \pi \in \operatorname{Obj} \mathscr{C}\right)_{\alpha}: \Phi \stackrel{t}{\rightarrow} \alpha \Phi .
$$

Это $\alpha$-преобразованиезадает тождественную стрелку для $\alpha$-функтора $\Phi$ как стрелку $i_{\Phi} \equiv\left(I_{\Phi}, \Phi, \Phi\right)_{\alpha}$.

Приведенные определения показьвают, что в рамках универсального класса $\alpha$ естественно определяются необходимые первичные понятия теории категорий. Отметим, что соответствующие определения без каких-либо изменений можно дать и в универсумах теорий $N B G$ или $M K M$.

Покажем теперь, как в рамках локальной теории множеств можно формализовать операции наивной теории категорий, известные как "категория категорий" и “категория функторов" (чего нельзя, как известно, сделать в $N B G$ и $M K M)$.

Поскольку нам потребуются “больши́е” совокупности $\alpha$-классов, не существующие в самом универсальном классе $\alpha$, возьмем любой “бо́льший” универсальный класс $\beta$, т.е. такой, что $\alpha \in \beta$. В силу аксиомы универсальности А6 это можно сделать.

Пользуясь тем, что аксиома $\alpha$-категории саt $\alpha(X)$ выписана в строгом и полном виде, легко убедиться, что аксиома саt $\alpha(X)$ является $\beta$-предикативной (точнее, может быть в силу $\alpha \in \beta$ приведена к $\beta$-предикативной форме). Поэтому в силу AS2 существует $\beta$-класс

$$
\operatorname{Cat}_{\alpha}^{\beta} \equiv\left\{X \in \beta \mid \operatorname{cat}_{\alpha}(X)\right\},
$$

состоящий из всех $\alpha$-категорий $\mathscr{C}$.

Аналогичным образом можно убедиться, что аксиома $\alpha$-функторной стрелки

$$
\operatorname{arr}_{\alpha}(U) \equiv(U \subset \alpha) \wedge \exists X, Y, Z\left(\operatorname{cat}_{\alpha}(X) \wedge \operatorname{cat}_{\alpha}(Y) \wedge Z \rightleftharpoons X \stackrel{f}{\rightarrow} Y \wedge U=(Z, X, Y)_{\alpha}\right)
$$

тоже является $\beta$-предикативной. Поэтому в силу AS2 существует $\beta$-класс

$$
f_{\operatorname{Arr}_{\alpha}^{\beta}}^{\beta} \equiv\left\{U \in \beta \mid \operatorname{arr}_{\alpha}(U)\right\}
$$

состоящий из всех $\alpha$-функторных стрелок $F \equiv(\Phi, \mathscr{C}, \mathscr{D})_{\alpha}$.

Если $\mathscr{C}$ и $\mathscr{D}-\alpha$-категории, то из $\alpha \in \beta$ и аксиомы подмножества А8 следует, что $\mathscr{C}$ и $\mathscr{D}$ являются $\beta$-множествами. Поэтому мы можем рассмотреть простую $\beta$-пару $(\mathscr{C}, \mathscr{D})_{\beta}$. Для такой пары также существует $\beta$-класс

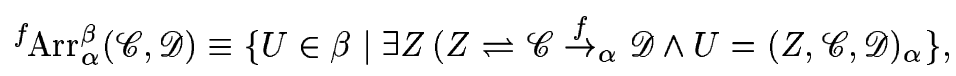

состоящий из всех $\alpha$-функторных стрелок $F \equiv(\Phi, \mathscr{C}, \mathscr{D})_{\alpha}$ из $\mathscr{C}$ в $\mathscr{D}$.

Используя этот $\beta$-класс и аксиомную схему AS2 мы можем корректно определить следуюшие $\beta$-классы:

$$
\begin{aligned}
\zeta \equiv & \left\{x \in \beta \mid \exists X, Y, U \in \beta\left(X, Y \in \mathrm{Cat}_{\alpha}^{\beta} \wedge U \in f_{\operatorname{Arr}_{\alpha}}^{\beta}(X, Y) \wedge x=\left\langle(X, Y)_{\beta}, U\right\rangle_{\beta}\right)\right\} \\
\subset & \left(\operatorname{Cat}_{\alpha}^{\beta} \times{ }_{\beta} \operatorname{Cat}_{\alpha}^{\beta}\right) *{ }_{\beta} f_{\operatorname{Arr}_{\alpha}}^{\beta}, \\
\eta \equiv & \left\{x \in \beta \mid \exists X, Y, Z, U, V, W \in \beta\left(X, Y, Z \in \operatorname{Cat}_{\alpha}^{\beta} \wedge U \in f_{\operatorname{Arr}_{\alpha}^{\beta}(X, Y)}\right.\right. \\
& \left.\left.\wedge V \in{ }^{f} \operatorname{Arr}_{\alpha}^{\beta}(Y, Z) \wedge W \in{ }^{f} \operatorname{Arr}_{\alpha}^{\beta}(X, Z) \wedge W=V \circ U \wedge x=\left\langle(U, V)_{\beta}, W\right\rangle_{\beta}\right)\right\} \\
\subset & \left({ }^{f} \operatorname{Arr}_{\alpha}^{\beta} \times{ }_{\beta}{ }^{f} \operatorname{Arr}_{\alpha}^{\beta}\right) *{ }_{\beta}{ }^{f} \operatorname{Arr}_{\alpha}^{\beta},
\end{aligned}
$$




$$
\begin{aligned}
& \vartheta \equiv\left\{x \in \beta \mid \exists X, U \in \beta\left(X \in \mathrm{Cat}_{\alpha}^{\beta} \wedge U \in f_{\operatorname{Arr}_{\alpha}^{\beta}}^{\beta}(X, X) \wedge U=i_{X} \wedge x=\langle X, U\rangle_{\beta}\right)\right\} \\
& \subset \mathrm{Cat}_{\alpha}^{\beta}{ }_{\beta}{ }^{f_{\mathrm{Arr}}}{ }_{\alpha}^{\beta} \text {. }
\end{aligned}
$$

Имея компоненты $\zeta, \eta$ и $\vartheta$, мы можем рассмотреть $\beta$-подкласс

$$
\begin{gathered}
s \equiv\left\{x \in \beta \mid \exists y, z \in \beta\left(y \in \Omega_{c} \wedge x=\langle y, z\rangle_{\beta} \wedge(y=\# \Longrightarrow z \in \zeta)\right.\right. \\
\wedge(y=\circ \Longrightarrow z \in \eta) \wedge(y=\leftrightarrow \Longrightarrow z \in \vartheta))\}
\end{gathered}
$$

$\beta$-класса $\Omega_{c} *_{\beta} \beta$.

Наконец, рассмотрим $\beta$-класс

$$
\mathbb{C}_{\alpha}^{\beta} \equiv\left(\left(\mathrm{Cat}_{\alpha}^{\beta},{ }^{f_{\mathrm{Arr}}}{ }_{\alpha}^{\beta}\right)_{\beta}, s\right)_{\beta}
$$

с носителем $A \equiv\left(\mathrm{Cat}_{\alpha}^{\beta}, f_{\mathrm{Arr}_{\alpha}}^{\beta}\right)_{\beta}$ и структурой $s$.

Теорема 1. $\beta$-класс $\mathbb{C}_{\alpha}^{\beta}$ является $\beta$-категорией.

ДокАЗАТЕЛЬСТво. Из определения следует, что $s$ является $\beta$-соответствием из $\Omega_{c}$ в $\beta$.

Проверим, что $s\langle \#\rangle=\zeta$. Пусть $A \in s\langle \#\rangle$. Это значит, что $\langle \#, A\rangle_{\beta} \in s$. По AS2 $\langle \#, A\rangle_{\beta}=\langle\omega, B\rangle_{\beta}$ для некоторых $\omega \in \Omega_{c}$ и $B \in \beta$. По следствию к лемме $2 \omega=\#$ и $A=B$. Снова применяя AS2, получаем $B \in \zeta$. Следовательно, $A \in \zeta$. Обратно, пусть $C \in \zeta \subset \beta$. Рассмотрим $\beta$-множество $r \equiv\langle \#, C\rangle_{\beta} \in \Omega_{c} *_{\beta} \beta$. По AS2 $r \in s$ и, значит, $C \in s\langle \#\rangle$.

Аналогично проверяется, что $s\langle 0\rangle=\eta$ и $s\langle\leftrightarrow\rangle=\vartheta$. Из этих равенств и определения $\beta$-классов $\zeta, \eta$ и $\vartheta$ следует, что свойство

$$
P_{c} 1 \equiv A_{c} 1\left(\mathrm{Cat}_{\alpha}^{\beta},{ }^{f} \mathrm{Arr}_{\alpha}^{\beta}, s\right)
$$

выполнено.

Проверим, что классы $L \equiv \zeta\left\langle(\mathscr{C}, \mathscr{D})_{\beta}\right\rangle$ и $R \equiv{ }^{f} \operatorname{Arr}_{\alpha}^{\beta}(\mathscr{C}, \mathscr{D})$ равны. Если $l \in L$, то $\left\langle(\mathscr{C}, \mathscr{D})_{\beta}, l\right\rangle_{\beta} \in \zeta$. Значит, по AS2 $\left\langle(\mathscr{C}, \mathscr{D})_{\beta}, l\right\rangle_{\beta}=\left\langle\left(\mathscr{C}^{\prime}, \mathscr{D}^{\prime}\right)_{\beta}, F\right\rangle_{\beta}$ для некоторых $\alpha$-категорий $\mathscr{C}^{\prime}$ и $\mathscr{D}^{\prime}$ и некоторой $\alpha$-функторной стрелки $F \equiv\left(\Phi, \mathscr{C}^{\prime}, \mathscr{D}^{\prime}\right)_{\alpha}$. По следствию к лемме $2(\mathscr{C}, \mathscr{D})_{\beta}=\left(\mathscr{C}^{\prime}, \mathscr{D}^{\prime}\right)_{\beta}$ и $l=F$. По лемме $5 \mathscr{C}=\mathscr{C}^{\prime}$ и $\mathscr{D}=\mathscr{D}^{\prime}$. Значит, $l \in R$. Обратно, если $r \in R$, то по $\mathrm{AS} 2\left\langle(\mathscr{C}, \mathscr{D})_{\beta}, r\right\rangle_{\beta} \in \zeta$. Значит, $r \in L$. Таким образом, $L=R$.

Из доказанного следует, что $s\langle \#\rangle\left\langle(\mathscr{C}, \mathscr{D})_{\beta}\right\rangle={ }^{f} \operatorname{Arr}_{\alpha}^{\beta}(\mathscr{C}, \mathscr{D})$.

Из определения ясно, что

$$
f_{\operatorname{Arr}_{\alpha}^{\beta}}^{\beta}=\cup\left({ }^{f} \operatorname{Arr}_{\alpha}^{\beta}(\mathscr{C}, \mathscr{D}) \mid(\mathscr{C}, \mathscr{D})_{\beta} \in \mathrm{Cat}_{\alpha}^{\beta} \times{ }_{\beta} \mathrm{Cat}_{\alpha}^{\beta}\right)_{\beta}
$$

Проверим, что классы $L \equiv \operatorname{rng}_{\beta} \zeta$ и $R \equiv f_{\operatorname{Arr}_{\alpha}}^{\beta}$ равны. Если $l \in L$, то $\langle p, l\rangle_{\beta} \in \zeta$ для некоторого $p \in \mathrm{Cat}_{\alpha}^{\beta} \times{ }_{\beta} \mathrm{Cat}_{\alpha}^{\beta}$. Значит, по $\mathrm{AS} 2\langle p, l\rangle_{\beta}=\left\langle(\mathscr{C}, \mathscr{D})_{\beta}, F\right\rangle_{\beta}$ для некоторых $\alpha$-категорий $\mathscr{C}$ и $\mathscr{D}$ и некоторой стрелки $F \equiv(\Phi, \mathscr{C}, \mathscr{D})_{\alpha}$. По следствию к лемме 2

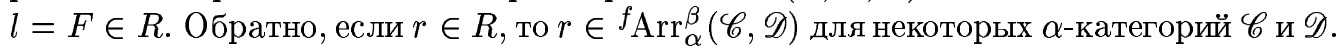
По AS2 $\left\langle(\mathscr{C}, \mathscr{D})_{\beta}, r\right\rangle_{\beta} \in \zeta$. Значит, $r \in L$. Таким образом, $L=R$.

Из доказанного следует, что $\operatorname{rng}_{\beta} s\langle \#\rangle={ }^{\mathrm{Arr}_{\alpha}}{ }_{\alpha}$.

Пусть $p \equiv(\mathscr{C}, \mathscr{D})_{\beta} \neq\left(\mathscr{C}^{\prime}, \mathscr{D}^{\prime}\right)_{\beta} \equiv q$ для некоторых $\alpha$-категорий $\mathscr{C}, \mathscr{C}^{\prime}, \mathscr{D}$ и $\mathscr{D}^{\prime}$. По следствию к лемме 2 или $\mathscr{C} \neq \mathscr{C}^{\prime}$, или $\mathscr{D} \neq \mathscr{D}^{\prime}$. Если $F \in s\langle \#\rangle\langle p\rangle={ }^{A_{A r r}}{ }_{\alpha}^{\beta}(\mathscr{C}, \mathscr{D})$ и 
$G \in s\langle \#\rangle\langle q\rangle={ }^{f} \operatorname{Arr}_{\alpha}^{\beta}\left(\mathscr{C}^{\prime}, \mathscr{D}^{\prime}\right)$, то $F=(\Phi, \mathscr{C}, \mathscr{D})_{\alpha}$ и $G=\left(\Psi, \mathscr{C}^{\prime}, \mathscr{D}^{\prime}\right)_{\alpha}$ для некоторых $\alpha$-функторов $\Phi: \mathscr{C} \stackrel{f}{\rightarrow}_{\alpha} \mathscr{D}$ и $\Psi: \mathscr{C}^{\prime} \stackrel{f}{\rightarrow}_{\alpha} \mathscr{D}^{\prime}$. Поэтому в любом случае по лемме $5 F \neq G$. Это означает, что $s\langle \#\rangle\langle p\rangle \cap s\langle \#\rangle\langle q\rangle=\varnothing$. Из доказанного следует, что свойство

$$
P_{c} 2 \equiv A_{c} 2\left(\mathrm{Cat}_{\alpha}^{\beta},{ }^{f} \operatorname{Arr}_{\alpha}^{\beta}, s\right)
$$

выполнено.

Проверим вьполнимость свойства

$$
P_{c} 3 \equiv A_{c} 3\left(\mathrm{Cat}_{\alpha}^{\beta},{ }^{f} \mathrm{Arr}_{\alpha}^{\beta}, s\right) .
$$

Если $l \in L \equiv \operatorname{dom}_{\beta} s\langle 0\rangle=\operatorname{dom}_{\beta} \eta$, то $\langle l, a\rangle_{\beta} \in \eta$ для некоторого $a \in{ }^{f} \operatorname{Arr}_{\alpha}^{\beta}$. Поэтому по AS2 $\langle l, a\rangle_{\beta}=\left\langle(F, G)_{\beta}, H\right\rangle_{\beta}$ для некоторых $\alpha$-категорий $\mathscr{C}, \mathscr{D}$ и $\mathscr{E}$ и некоторых $\alpha$-функторных стрелок $F \equiv(\Phi, \mathscr{C}, \mathscr{D})_{\alpha}, G \equiv(\Psi, \mathscr{D}, \mathscr{E})_{\alpha}$ и $H \equiv(\Omega, \mathscr{C}, \mathscr{E})_{\alpha}$ таких, что $H=G \circ F$. По следствию к лемме $2 l=(F, G)_{\beta}$ и $a=H$. Как установлено вьше, $F \in s\langle \#\rangle\left\langle(\mathscr{C}, \mathscr{D})_{\beta}\right\rangle$ и $G \in s\langle \#\rangle\left\langle(\mathscr{D}, \mathscr{E})_{\beta}\right\rangle$.

Это значит, что $H \in s\langle 0\rangle\langle l\rangle$ и

$$
\begin{aligned}
l \in\{ & x \in \alpha \mid \exists x^{\prime}, y^{\prime}, z^{\prime}, y^{\prime \prime}, z^{\prime \prime} \in \alpha\left(x^{\prime}, y^{\prime}, z^{\prime} \in \mathrm{Cat}_{\alpha}^{\beta}\right. \\
& \wedge y^{\prime \prime}, z^{\prime \prime} \in{ }^{f_{1 r r}} \operatorname{Ar}_{\alpha} \wedge\left(y^{\prime \prime} \in s\langle \#\rangle\left\langle\left(x^{\prime}, y^{\prime}\right)_{\beta}\right\rangle\right) \\
& \left.\left.\wedge\left(z^{\prime \prime} \in s\langle \#\rangle\left\langle\left(y^{\prime}, z^{\prime}\right)_{\beta}\right\rangle\right) \wedge\left(x=\left(y^{\prime \prime}, z^{\prime \prime}\right)_{\beta}\right)\right)\right\} \equiv R .
\end{aligned}
$$

Обратно, если $r \in R$, то $r=(F, G)_{\beta}$ для некоторьгх $\alpha$-категорий $\mathscr{C}, \mathscr{D}$ и $\mathscr{E}$ и некоторых $\alpha$-функторных стрелок $F \in s\langle \#\rangle\left\langle(\mathscr{C}, \mathscr{D})_{\beta}\right\rangle={ }_{\mathrm{Arr}_{\alpha}}^{\beta}(\mathscr{C}, \mathscr{D})$ и $G \in s\langle \#\rangle\left\langle(\mathscr{D}, \mathscr{E})_{\beta}\right\rangle=$ ${ }^{\mathrm{Arr}_{\alpha}^{\beta}}(\mathscr{D}, \mathscr{E})$. Рассмотрим $\alpha$-функторную стрелку $H \equiv G \circ F \in{ }^{f} \operatorname{Arr}_{\alpha}^{\beta}(\mathscr{C}, \mathscr{E})$. По AS2 $\langle r, H\rangle_{\beta}=\left\langle(F, G)_{\beta}, H\right\rangle_{\beta} \in \eta=s\langle$ о $\rangle$. Значит, $r \in L$. Таким образом, $L=R$.

Проверим теперь, что соответствие $s\langle 0\rangle$ является однозначньм. Пусть $l \in L$ и $a, a^{\prime} \in$ $s\left\langle\right.$ о $\langle l\rangle=\eta\langle l\rangle$. Тогда $\langle l, a\rangle_{\beta} \in \eta$ и $\left\langle l, a^{\prime}\right\rangle_{\beta} \in \eta$. По AS2 $\langle l, a\rangle_{\beta}=\left\langle(F, G)_{\beta}, H\right\rangle_{\beta}$ и $\left\langle l, a^{\prime}\right\rangle_{\beta}=\left\langle\left(F^{\prime}, G^{\prime}\right)_{\beta}, H^{\prime}\right\rangle_{\beta}$ для некоторых $\alpha$-категорий $\mathscr{C}, \mathscr{D}, \mathscr{E}, \mathscr{C}^{\prime}, \mathscr{D}^{\prime}$ и $\mathscr{E}^{\prime}$ и некоторых $\alpha$-функторных стрелок $F \equiv(\Phi, \mathscr{C}, \mathscr{D})_{\alpha}, G \equiv(\Psi, \mathscr{D}, \mathscr{E})_{\alpha}, H \equiv(\Omega, \mathscr{C}, \mathscr{E})_{\alpha}, F^{\prime} \equiv$ $\left(\Phi^{\prime}, \mathscr{C}^{\prime}, \mathscr{D}^{\prime}\right)_{\alpha}, G^{\prime} \equiv\left(\Psi^{\prime}, \mathscr{D}^{\prime}, \mathscr{E}^{\prime}\right)_{\alpha}$ и $H^{\prime} \equiv\left(\Omega^{\prime}, \mathscr{C}^{\prime}, \mathscr{E}^{\prime}\right)_{\alpha}$ таких, что $H \equiv G$ о $F$ и $H^{\prime} \equiv G^{\prime} \circ F^{\prime}$. По следствию к лемме $2 l=(F, G)_{\beta}, l=\left(F^{\prime}, G^{\prime}\right)_{\beta}, a=H$ и $a^{\prime}=H^{\prime}$. По лемме $5 F=F^{\prime}$ и $G=G^{\prime}$. Значит, $a=H=G \circ F=G^{\prime} \circ F^{\prime}=H^{\prime}=a^{\prime}$. Таким образом, $s\langle$ о $\rangle$ является $\alpha$-отображением из $\operatorname{dom}_{\alpha} s\langle\circ\rangle{ }_{\text {в }} \operatorname{Arr}_{\alpha}^{\beta}$. Это заканчивает проверку справедливости свойства $P_{c} 3$. Более того, мы получаем равенство

$$
s\langle\circ\rangle\left((F, G)_{\beta}\right)=s\langle\circ\rangle(l)=H=G \circ F .
$$

Проверим справедливость свойства

$$
P_{c} 4 \equiv A_{c} 4\left(\mathrm{Cat}_{\alpha}^{\beta},{ }_{\mathrm{Arr}_{\alpha}}^{\beta}, s\right) .
$$

Если $l \in L \equiv \operatorname{dom}_{\beta} s\langle\leftrightarrow\rangle=\operatorname{dom}_{\beta} \vartheta$, то $\langle l, a\rangle_{\beta} \in \vartheta$ для некоторого $a \in f_{A} r_{\alpha}^{\beta}$. Поэтому по AS2 $\langle l, a\rangle_{\beta}=\left\langle\mathscr{C}, i_{\mathscr{C}}\right\rangle_{\beta}$ для некоторой $\alpha$-категории $\mathscr{C}$. По следствию к лемме 2 $l=\mathscr{C} \in \mathrm{Cat}_{\alpha}^{\beta} \equiv R$. Обратно, если $\mathscr{C} \in R$, то по $\mathrm{AS} 2\left\langle\mathscr{C}, i_{\mathscr{C}}\right\rangle_{\beta} \in \vartheta$. Следовательно, $\mathscr{C} \in \operatorname{dom}_{\beta} \vartheta=L$. Это означает, что $L=R$, т.е. $\beta$-соответствие $s\langle\leftrightarrow\rangle$ является тотальным. 
Пусть $l \in L$ и $a, a^{\prime} \in s\langle\leftrightarrow\rangle\langle l\rangle=\vartheta\langle l\rangle$. Тогда $\langle l, a\rangle_{\beta} \in \vartheta$ и $\left\langle l, a^{\prime}\right\rangle_{\beta} \in \vartheta$. По AS2 $\langle l, a\rangle_{\beta}=\left\langle\mathscr{C}, i_{\mathscr{C}}\right\rangle_{\beta}$ и $\left\langle l, a^{\prime}\right\rangle=\left\langle\mathscr{C}^{\prime}, i_{\mathscr{C}}\right\rangle_{\beta}$ для некоторых $\alpha$-категорий $\mathscr{C}$ и $\mathscr{C}^{\prime}$. По следствию к лемме $2 l=\mathscr{C}, a=i_{\mathscr{C}}, l=\mathscr{C}^{\prime}$ и $a^{\prime}=i_{\mathscr{C}}$. Следовательно, $a=a^{\prime}$, т.е. $\beta$-соответствие $s\langle\leftrightarrow\rangle$ является однозначным. Значит, оно является $\beta$-отображением из $\mathrm{Cat}_{\alpha}^{\beta}$ в ${ }^{f} \mathrm{Arr}_{\alpha}^{\beta}$ таким, что

$$
s\langle\leftrightarrow\rangle(\mathscr{C})=i_{\mathscr{C}} \in{ }^{f} \operatorname{Arr}_{\alpha}^{\beta}(\mathscr{C}, \mathscr{C})=s\langle \#\rangle\left\langle(\mathscr{C}, \mathscr{C})_{\beta}\right\rangle .
$$

Это заканчивает проверку справедливости свойства $P_{c} 4$.

Пусть

$$
l \in s\langle 0\rangle\left[s\langle \#\rangle\left\langle(\mathscr{C}, \mathscr{D})_{\beta}\right\rangle \times_{\beta} s\langle \#\rangle\left\langle(\mathscr{D}, \mathscr{E})_{\beta}\right\rangle\right] .
$$

Тогда по AS2 $\left\langle(a, b)_{\beta}, l\right\rangle_{\beta} \in s\langle$ о $\rangle=\eta$ для некоторых элементов $a \in s\langle \#\rangle\left\langle(\mathscr{C}, \mathscr{D})_{\beta}\right\rangle=$

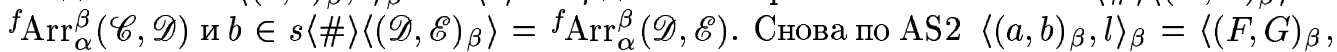
$H\rangle_{\beta}$ для некоторых $\alpha$-категорий $\mathscr{C}, \mathscr{D}$ и $\mathscr{E}$ и некоторых $\alpha$-функторных стрелок $F \equiv$ $(\Phi, \mathscr{C}, \mathscr{D})_{\alpha}, G \equiv(\Psi, \mathscr{D}, \mathscr{E})_{\alpha}$ и $H \equiv(\Omega, \mathscr{C}, \mathscr{E})_{\alpha}$ таких, что $H=G \circ F$. По следствию к лемме $2 l=H \in{ }^{f} \operatorname{Arr}_{\alpha}^{\beta}(\mathscr{C}, \mathscr{E})=s\langle \#\rangle\left\langle(\mathscr{C}, \mathscr{E})_{\beta}\right\rangle$. Это доказывает свойство

$$
P_{c} 5 \equiv A_{c} 5\left(\mathrm{Cat}_{\alpha}^{\beta},{ }^{f} \operatorname{Arr}_{\alpha}^{\beta}, s\right) .
$$

Пусть $\mathscr{C}, \mathscr{D}, \mathscr{E}, F-\alpha$-категории и $F, G, H$ - стрелки такие, что

$$
\begin{aligned}
& F \in s\langle \#\rangle\left\langle(\mathscr{C}, \mathscr{D})_{\beta}\right\rangle={ }^{f_{A r r}}{ }_{\alpha}(\mathscr{C}, \mathscr{D}), \\
& G \in s\langle \#\rangle\left\langle(\mathscr{D}, \mathscr{E})_{\beta}\right\rangle={ }^{f_{A r r}}{ }_{\alpha}(\mathscr{D}, \mathscr{E}), \\
& H \in s\langle \#\rangle\left\langle(\mathscr{E}, \mathscr{F})_{\beta}\right\rangle={ }^{f} \operatorname{Arr}_{\alpha}^{\beta}(\mathscr{E}, \mathscr{F}) \text {. }
\end{aligned}
$$

Тогда

$$
\begin{aligned}
s\langle\circ\rangle\left(\left(s\langle\circ\rangle\left((F, G)_{\beta}\right), H\right)_{\beta}\right) & =H \circ s\langle\circ\rangle\left((F, G)_{\beta}\right)=H \circ(G \circ F) \\
& =(H \circ G) \circ F=s\langle\circ\rangle\left((G, H)_{\beta}\right) \circ F \\
& =s\langle\circ\rangle\left(\left(F, s\langle\circ\rangle\left((G, H)_{\beta}\right)\right)_{\beta}\right) .
\end{aligned}
$$

Отсюда следует свойство

$$
P_{c} 6 \equiv A_{c} 6\left(\mathrm{Cat}_{\alpha}^{\beta},{ }^{f} \operatorname{Arr}_{\alpha}^{\beta}, s\right) .
$$

Пусть $\mathscr{C}, \mathscr{D}, \mathscr{E}-\alpha$-категории и $F, G-$ стрелки такие, что

$$
F \in s\langle \#\rangle\left\langle(\mathscr{D}, \mathscr{C})_{\beta}\right\rangle={ }^{f} \operatorname{Arr}_{\alpha}^{\beta}(\mathscr{D}, \mathscr{C}) \quad \text { и } \quad G \in s\langle \#\rangle\left\langle(\mathscr{C}, \mathscr{E})_{\beta}\right\rangle={ }^{f} \operatorname{Arr}_{\alpha}^{\beta}(\mathscr{C}, \mathscr{E}) .
$$

Тогда

$$
s\langle\circ\rangle\left((F, s\langle\leftrightarrow\rangle(\mathscr{C}))_{\beta}\right)=i_{\mathscr{C}} \circ F=F \quad \text { и } \quad s\langle\circ\rangle\left((s\langle\leftrightarrow\rangle(\mathscr{C}), G)_{\beta}\right)=G \circ i_{\mathscr{C}}=G .
$$

Отсюда следует свойство

$$
P_{c} 7 \equiv A_{c} 7\left(\mathrm{Cat}_{\alpha}^{\beta},{ }_{\mathrm{Arr}_{\alpha}}^{\beta}, s\right)
$$

Теорема доказана.

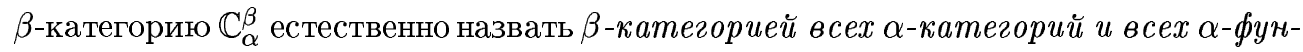
кторных стрелок между ними. 
Пусть теперь $\mathscr{C}$ и $\mathscr{D}$ - фиксированные $\alpha$-категории. Формулу

$$
X \rightleftharpoons \mathscr{C} \stackrel{f}{\rightarrow}_{\alpha} \mathscr{D}
$$

будем назьвать аксиомой $\alpha$-функтора из $\alpha$-категории $\mathscr{C}$ в $\alpha$-категорию $\mathscr{D}$ (со свободной переменной $X$ ). Так же, как и вьше, можно убедиться, что эта аксиома является $\beta$-предикативной. Поэтому в силу $\mathrm{AS} 2$ существует $\beta$-класс

$$
\text { Funct }_{\alpha}^{\beta}(\mathscr{C}, \mathscr{D}) \equiv\left\{X \in \beta \mid X \rightleftharpoons \mathscr{C} \stackrel{f}{\rightarrow}_{\alpha} \mathscr{D}\right\},
$$

состоящий из всех $\alpha$-функторов из $\alpha$-категории $\mathscr{C}$ в $\alpha$-категорию $\mathscr{D}$.

Аналогично аксиома $\alpha$-преобразовательной стрелки

$$
\begin{aligned}
{ }^{t} \operatorname{arr}_{\alpha}(U) \equiv & (U \subset \alpha) \wedge \exists X, Y, Z\left(X \rightleftharpoons \mathscr{C} \stackrel{f}{\rightarrow}_{\alpha} \mathscr{D}\right. \\
& \left.\wedge Y \rightleftharpoons \mathscr{C} \stackrel{f}{\rightarrow}_{\alpha} \mathscr{D} \wedge Z \rightleftharpoons X \stackrel{t}{\rightarrow}_{\alpha} Y \wedge U=(Z, X, Y)_{\alpha}\right)
\end{aligned}
$$

тоже является $\beta$-предикативной. Поэтому в силу $\mathrm{AS} 2$ существует $\beta$-класс

$$
{ }^{t} \operatorname{Arr}_{\alpha}^{\beta} \equiv\left\{U \in \beta \mid{ }^{t} \operatorname{Arr}_{\alpha}(U)\right\},
$$

состоящий из всех $\alpha$-преобразовательных стрелок $F \equiv(T, \Phi, \Psi)_{\alpha}$.

Если $\Phi$ и $\Psi-\alpha$-функторы из $\mathscr{C}$ в $\mathscr{D}$, то из $\alpha \in \beta$ и аксиомы подмножества А 8 следует, что $\Phi$ и $\Psi$ являются $\beta$-множествами. Поэтому можно рассмотреть простую $\beta$-пару $(\Phi, \Psi)_{\beta}$. Для такой пары также существует $\beta$-класс

$$
{ }^{t} \operatorname{Arr}_{\alpha}^{\beta}(\Phi, \Psi) \equiv\left\{U \in \beta \mid \exists Z\left(Z \rightleftharpoons \Phi \stackrel{t}{\rightarrow} \alpha \wedge U=(Z, \Phi, \Psi)_{\alpha}\right)\right\},
$$

состоящий из всех $\alpha$-преобразовательных стрелок $F \equiv(T, \Phi, \Psi)_{\alpha}$.

Используя введенные $\beta$-классы и аксиомную схему AS2, мы можем корректно определить следующие $\beta$-классы:

$$
\begin{aligned}
\zeta \equiv & \left\{x \in \beta \mid \exists X, Y, U \in \beta\left(X, Y \in \operatorname{Funct}_{\alpha}^{\beta}(\mathscr{C}, \mathscr{D})\right.\right. \\
& \left.\left.\wedge U \in{ }^{t} \operatorname{Arr}_{\alpha}^{\beta}(X, Y) \wedge x=\left\langle(X, Y)_{\beta}, U\right\rangle_{\beta}\right)\right\} \\
\subset & \left(\operatorname{Funct}_{\alpha}^{\beta}(\mathscr{C}, \mathscr{D}) \times{ }_{\beta} \operatorname{Funct}_{\alpha}^{\beta}(\mathscr{C}, \mathscr{D})\right) *_{\beta}{ }^{t} \operatorname{Arr}_{\alpha}^{\beta} ; \\
\eta \equiv & \left\{x \in \beta \mid \exists X, Y, Z, U, V, W \in \beta\left(X, Y, Z \in \operatorname{Funct}_{\alpha}^{\beta}(\mathscr{C}, \mathscr{D})\right.\right. \\
& \wedge U \in{ }^{t} \operatorname{Arr}_{\alpha}^{\beta}(X, Y) \wedge V \in{ }^{t} \operatorname{Arr}_{\alpha}^{\beta}(Y, Z) \\
& \left.\left.\wedge W \in{ }^{t} \operatorname{Arr}_{\alpha}^{\beta}(X, Z) \wedge W=V \circ U \wedge x=\left\langle(U, V)_{\beta}, W\right\rangle_{\beta}\right)\right\} \\
\subset & \left({ }^{t} \operatorname{Arr}_{\alpha}^{\beta} \times{ }_{\beta}{ }^{t} \operatorname{Arr}_{\alpha}^{\beta}\right) *_{\beta}{ }^{t} \operatorname{Arr}_{\alpha}^{\beta} ; \\
\vartheta \equiv & \left\{x \in \beta \mid \exists X, U \in \beta\left(X \in \operatorname{Funct}_{\alpha}^{\beta}(\mathscr{C}, \mathscr{D})\right.\right. \\
& \left.\left.\wedge U \in{ }^{t} \operatorname{Arr}_{\alpha}^{\beta}(X, X) \wedge U=i_{X} \wedge x=\langle X, U\rangle_{\beta}\right)\right\} \\
\subset & \operatorname{Funct}_{\alpha}^{\beta}(\mathscr{C}, \mathscr{D}) *{ }_{\beta}{ }^{t} \operatorname{Arr}_{\alpha}^{\beta} .
\end{aligned}
$$

Имея компоненты $\zeta, \eta$ и $\vartheta$, рассмотрим $\beta$-класс $s$, определенный перед теоремой 1.

Наконец, рассмотрим $\beta$-класс

$$
\mathbb{F}_{\alpha}^{\beta}(\mathscr{C}, \mathscr{D}) \equiv\left(\left(\text { Funct }_{\alpha}^{\beta}(\mathscr{C}, \mathscr{D}),{ }^{t} \operatorname{Arr}_{\alpha}^{\beta}\right)_{\beta}, s\right)_{\beta}
$$

с носителем $A \equiv\left(\text { Funct }{ }_{\alpha}^{\beta}(\mathscr{C}, \mathscr{D}),{ }^{t} \operatorname{Arr}_{\alpha}^{\beta}\right)_{\beta}$ и структурой $s$. 
ТЕОрема 2. $\beta$-класс $\mathbb{F}_{\alpha}^{\beta}(\mathscr{C}, \mathscr{D})$ является $\beta$-категорией.

ДоКАЗАТЕЛЬСТВО проводится полностью по той же схеме, что и доказательство теоремы 1 , и мы не будем его здесь приводить.

$\beta$-категорию $\mathbb{F}_{\alpha}^{\beta}(\mathscr{C}, \mathscr{D})$ естественно назвать $\beta$-категорией всех $\alpha$-функторов из $\alpha$ категории $\mathscr{C}$ в $\alpha$-категорию $\mathscr{D}$ и всех $\alpha$-преобразовательных стрелок между ними.

Конструкции $\mathbb{C}_{\alpha}^{\beta}$ и $\mathbb{F}_{\alpha}^{\beta}(\mathscr{C}, \mathscr{D})$ показьвают, что понятие $\alpha$-категории является замкнутым относительно таких важных операций наивной теории категорий, как "категория категорий” и “категория функторов".

\section{СПИСОК ЦИТИРОВАННОЙ ЛИТЕРАТУРЫ}

[1] Куратовский К., Мостовский А. Теория множеств. М.: Мир, 1970.

[2] Келли Дж. Л. Общая топология. М.: Наука, 1968.

[3] Мендельсон Э. Введение в математическую логику. М.: Наука, 1971.

[4] Eilenberg S., MacLane S. General theory of natural equivalences // Trans. Amer. Math. Soc. 1945. V. 58. P. 231-294.

[5] MacLane S. Locally small categories and the foundations of set theory // Symposium. Warsaw 1959. Oxford: Pergamon, 1961. P. 25-43.

[6] Ehresmann C. Guttungen von lokalen Strukturen // J. -ber. Deutsch. Math. Verein. 1957. V. 60. № 2. P. 49-77.

[7] Dedecker P. Introduction aux structures locales // Coll. Géom. Diff. Glob. Bruxelles. 1958. P. 103-135.

[8] Sonner J. The formal definition of categories // Math. Z. 1962. V. 80. P. 163-176.

[9] Gabriel P. Des catégories abéliennes // Bull. Soc. Math. France. 1962. V. 90. P. 323-448.

[10] da Costa N. On two systems of set theory // Proc. Koninkl. Nederl. Akad. Wet. Ser. A. 1965. V. 68. №1. P. 95-98.

[11] da Costa N. Two formal systems of set theory // Proc. Koninkl. Nederl. Akad. Wet. Ser. A. 1967. V. 70. № 1. P. 45-51.

[12] Isbell J. Structure of categories // Bull. Amer. Math. Soc. 1966. V. 72. P. 619-655.

[13] MacLane S. One universe as a foundation for category theory // Lecture Notes in Math. 1969. V. 106. P. $192-200$.

[14] MacLane S. Categories for Working Mathematician. Berlin: Springer-Verlag, 1971.

[15] Feferman S. Set-theoretical foundations of category theory // Lecture Notes in Math. 1969. V. 106. P. 201-247.

[16] Herrlich H., Strecker G. Category Theory. An Introduction. Berlin: Heldermann Verlag, 1979.

[17] Захаров В. К., Михалёв А. В. Локальная теория классов и множеств как основание для теории категорий // Математические методы и приложения. Tp. IX математических чтений МГСУ. Москва: МГСУ, 2002. С. 91-94.

[18] Андреев П. В., Бунина Е.И., Захаров В. К. Михалёв А. В. Основания теории категорий в рамках локальной теории множеств // Тезисы докладов международной алгебраической конференции. Санкт-Петербург: ПОМИ, 2002. С. 10-11.

[19] Hatcher W. S. The Logical Foundations of Mathematics. Oxford: Pergamon Press, 1982.

Центр новых информационных технологий

Поступило

Московского государственного университета им. М. В. Ломоносова

E-mail: valeri@zaharov.mccme.ru

16.12.2002

Исправленный вариант 18.02.2004 